\title{
High-Efficiency Fluorescence through Bioinspired Supramolecular Self-Assembly
}

Yu Chen ${ }^{1}$, Asuka A. Orr ${ }^{2}$, Kai Tao $^{1}$, Zhibin Wang ${ }^{3}$, Antonella Ruggiero ${ }^{4}$, Linda J. W. Shimon ${ }^{5}$, Lee Schnaider $^{1}$, Alicia Goodall ${ }^{2}$, Sigal Rencus-Lazar ${ }^{1}$, Sharon Gilead ${ }^{1}$, Inna Slutsky ${ }^{4}$, Phanourios Tamamis $^{2 *}$, Zhan'ao Tan ${ }^{*}$, Ehud Gazit ${ }^{*}$

${ }^{1}$ Department of Molecular Microbiology and Biotechnology, George S. Wise Faculty of Life Sciences, Tel Aviv University, 6997801, Tel Aviv, Israel

${ }^{2}$ Artie McFerrin Department of Chemical Engineering, Texas A\&M University, College Station, 77843-3122, Texas, United States

${ }^{3}$ State Key Laboratory of Alternate Electrical Power System with Renewable Energy Sources, North China Electric Power University, Beijing 102206, China

${ }^{4}$ Department of Physiology and Pharmacology, Sackler Faculty of Medicine, Tel Aviv University, Tel Aviv University, 6997801, Tel Aviv, Israel

${ }^{5}$ Department of Chemical Research Support, Weizmann Institute of Science, 76100, Rehovot, Israel ${ }^{6}$ Beijing Advanced Innovation Center for Soft Matter Science and Engineering, Beijing University of Chemical Technology, 100029, Beijing, China

\section{Supporting Information}




\section{Table of Contents}

- Experimental Section and Materials

- Supplementary Figures

- Supplementary Tables

- References

- Additional Supporting Information includes three movies: Movie S1, Movie S2, Movie S3, and three crystallographic data: crystallographic data S1-CHH-Zn(II), crystallographic data S2-CHH-NaNO${ }_{3}$, crystallographic data S3-CHH-Zn( $\left(\mathrm{NO}_{3}\right)_{2}$ 


\section{Experimental Section and Materials}

1. Materials. $\mathrm{CHH}$ was purchased from GL Biochem (Shanghai, China). Zinc nitrate $\left(\mathrm{Zn}\left(\mathrm{NO}_{3}\right)_{2}\right)$, zinc chloride $\left(\mathrm{ZnCl}_{2}\right)$, zinc bromide $\left(\mathrm{ZnBr}_{2}\right)$, zinc iodide $\left(\mathrm{ZnI}_{2}\right)$, sodium nitrate $\left(\mathrm{NaNO}_{3}\right)$, polyvinyl pyrrolidone (PVP), 1-methyl-2-pyrrolidone (NMP), dimethylformamide (DMF), dimethyl sulfoxide (DMSO), isopropanol, ethanol were purchased from Sigma Aldrich (Rehovot, Israel). Poly(3,4-ethylenedioxythiophene): poly(styrenesulfonate) (PEDOT: PSS) (Clevios P VP AI 4083) was purchased from H. C. Stark. Poly (N-vinyl carbazole) (PVK) was purchased from Tokyo chemical industry Co. Ltd. 1,3,5-tris(N-phenylbenzimidazol-2-yl) benzene (TPBI) was purchased from Xi'an Polymer Light Technology Corp. PEO (M.W. = 1000 000). Epirubicin hydrochloride (EPI) was purchased from Glentham life science, and DRAQ5 was purchased from Biolegend Inc. All materials were used as received without further purification. Water was processed using a Millipore purification system (Darmstadt, Germany) with minimum resistivity of $18.2 \mathrm{M} \Omega \mathrm{cm} .450$ $\mathrm{nm}$ emissive $\mathrm{InGaN}$ chip (emission peak at $450 \mathrm{~nm}$, operation under voltage of $3.0 \mathrm{~V}$ ) was purchased from Greatshine Semiconductor Technology Co. Ltd.

2. Peptide self-assembly. Fresh stock solutions of $\mathrm{CHH}$ were prepared by dissolving the peptide into $5 \%(\mathrm{v} / \mathrm{v}) \mathrm{DMF} /$ isopropanol at a concentration of $0.02 \mathrm{mmol} \mathrm{mL}^{-1}$. For assembly, $2.97 \mathrm{mg}$ of metal salt $\mathrm{Zn}\left(\mathrm{NO}_{3}\right)_{2}$ was added into the peptide solution under vigorous sonication, and the colorless solution was incubated in an $80^{\circ} \mathrm{C}$ water bath for 1 hour. The color of the solution will subsequently turned into light yellow.

3. Crystal preparation. (1) $\mathrm{CHH}-\mathrm{ZnI}_{2}$ : the weighed $\mathrm{CHH}$ powder was dissolved in $5 \%(\mathrm{v} / \mathrm{v})$ $\mathrm{DMF} /$ isopropanol, to a concentration of $5.48 \mathrm{mg} \mathrm{mL}^{-1}, 6.38 \mathrm{mg}$ of metal salt $\mathrm{ZnI}_{2}$ was added under vigorous sonication, followed by a 1 hour incubation at $80^{\circ} \mathrm{C}$ and filtration through a $0.45 \mu \mathrm{m}$ PTFE membrane (Merck Millipore, Carrigtwohill, Ireland). Red rod crystals appeared after ten days and reached a maximum size after 30 days. (2) $\mathrm{CHH}-\mathrm{NaNO}_{3}$ : the weighed $\mathrm{CHH}$ powder was dissolved into $5 \%(\mathrm{v} / \mathrm{v}) \mathrm{DMF} / \mathrm{ethanol}$, to a concentration of $5.48 \mathrm{mg} \mathrm{mL}^{-1}$, and $1.7 \mathrm{mg}$ of metal salt $\mathrm{NaNO}_{3}$ was added under vigorous sonication, followed by a 1 hour incubation at $80^{\circ} \mathrm{C}$ and filtration through a $0.45 \mu \mathrm{m}$ PTFE membrane (Merck Millipore, Carrigtwohill, Ireland). Colorless plaque-shaped crystals appeared after five days and reached a maximum size after 30 days. (3) $\mathrm{CHH}-\mathrm{Zn}\left(\mathrm{NO}_{3}\right)_{2}$ : 
the weighed CHH powder was dissolved in 5\% (v/v) DMF/ethanol, to a concentration of $5.48 \mathrm{mg}$ $\mathrm{mL}^{-1}$, and $2.97 \mathrm{mg}$ of metal salt $\mathrm{Zn}\left(\mathrm{NO}_{3}\right)_{2}$ was added under vigorous sonication, followed by a 1 hour incubation at $80^{\circ} \mathrm{C}$. Yellow needle-shaped crystals appeared after 15 mins and reached a maximum size within 2 hours.

\section{Characterization}

Atomic force microscopy (AFM). $5 \mu \mathrm{L}$ of sample solution was dropped onto a freshly cleaved mica surface and dried by $\mathrm{N}_{2}$ purge $(99.99 \%)$. The mica was then rinsed with water and gently purge dried with nitrogen. A topographic image was recorded under a Dimension icon AFM (Bruker) in the tapping mode at ambient temperature, with a $512 \times 512$-pixel resolution and a scanning speed of $1.0 \mathrm{~Hz}$. Nanoscope Analysis software was used for data collection and analysis.

Transmission electron microscopy (TEM). Aliquots $(10 \mu \mathrm{L})$ of CHH-Zn were added into a glow discharge copper grid (400 mesh) coated with a thin carbon film for 2 min. Excess solution was then removed, and the grid was washed three times with deionized water. TEM images were viewed using a FEI Tecnai F20 electron microscope operating at $80 \mathrm{kV}$.

Dynamic light scattering (DLS). $850 \mu \mathrm{L}$ of the sample solution was introduced into a DTS1070 folded capillary cell (Malvern, Worcestershire, U.K.), and the size was measured using a Zetasizer Nano ZS analyzer (Malvern Instruments, Malvern, UK) at $25.0^{\circ} \mathrm{C}$ and a backscatter detector $\left(173^{\circ}\right)$. Three measurements were performed and averaged for accuracy.

UV-Vis spectra. UV-Vis spectra between 200 to $800 \mathrm{~nm}$ were recorded on an Agilent Cary 100 UV-Vis spectrophotometer with a quartz cuvette of $1 \mathrm{~mm}$ path length.

Mass spectrometry (MS). The $\mathrm{CHH}-\mathrm{Zn}$ sample, along with the untreated $\mathrm{CHH}$, were dissolved into a $1 \%(\mathrm{v} / \mathrm{v})$ trifluoroacetic acid/water mixture. The MS experiment was performed using a LCMS Xevo-TQD system including an Acquity model UPLC and a triple quad mass spectrometer (Waters, Massachusetts, USA). The positive electrospray ionization $\left(\mathrm{ES}^{+}\right)$channel was used for analysis.

X-ray crystallography. Crystals suitable for diffraction were coated with Paratone oil (Hampton Research), mounted on loops and flash frozen in liquid nitrogen. Single crystal X-ray diffraction data measurement was performed using a Rigaku XtaLab ${ }^{\text {Pro }}$ system with $\mathrm{CuK}_{\alpha 1}(\lambda=1.5418 \AA)$ 
radiation at 100(2) K. Data were collected and processed using CrysAlisPro 1.171.39.22a (Rigaku OD, 2015). The structure was solved by direct methods using SHELXT-2016/4 and refined by fullmatrix least squares against F2 with SHELXL-2013.

Powder X-ray diffraction (PXRD). Self-assemblies of CHH-Zn( $\left(\mathrm{NO}_{3}\right)_{2}$ was centrifuged at 15000 rpm for $20 \mathrm{~min}$ and the precipitates were washed three times with Milli-Q water, flash-frozen immediately, and lyophilized for $72 \mathrm{~h}$. The resulting powder samples were deposited on a quartz zero-background sample holder. The diffraction patterns were collected using a D8ADVANCE diffractometer (Bruker, Germany) equipped with a linear detector LYNXEYE XE. Data collection was performed at room temperature with a scan range $2 \theta$ of $5-45^{\circ}$.

Fluorescence lifetime microscopy (FLIM). Fluorescence lifetime imaging was acquired using an LSM 7 MP two-photon microscope (Carl Zeiss, Weimar, Germany) coupled to the Becker and Hickl (BH) simple-Tau-152 system. Chameleon Ti: Sapphire laser system with $80 \mathrm{MHz}$ repetition rate was used to excite the sample at $900 \mathrm{~nm}$. Images were acquired using a Zeiss $20 \mathrm{x} 1 \mathrm{NA}$ waterimmersion objective. A Zeiss dichroic mirror (LP 760) was used to separate the excitation and emission light wavelengths. Emission light was collected via a hybrid GaAsP detector (HPM-10040, BH, Berlin, Germany) with a GFP bandpass filter. The image acquisition time was $60 \mathrm{sec}$. in order to collect a sufficient number of photons.

Fluorescence spectroscopy and quantum yield (QY) measurement. $600 \mu \mathrm{L}$ sample solution was pipetted into a $1.0 \mathrm{~cm}$ path-length quartz cuvette, and the spectrum was collected using a FluoroMax-4 Spectrofluorometer (Horiba Jobin Yvon, Kyoto, Japan) at ambient temperature. The excitation and emission wavelengths were set at $300-500 \mathrm{~nm}$ and $400-650 \mathrm{~nm}$, respectively, with a slit of $2 \mathrm{~nm}$. Absolute fluorescence QY measurements were performed using Quanta-Phi integrating sphere connected to Fluoromex-4.

Nuclear magnetic resonance (NMR). $\mathrm{CHH}-\mathrm{Zn}\left(\mathrm{NO}_{3}\right)_{2}$ were dissolved in isopropanol-d8 with tetramethylsilane (TMS) as the internal standard. ${ }^{1} \mathrm{H}$ NMR spectra were recorded on a Bruker AV400 NMR spectrometer with chemical shifts reported as ppm. The difference in the chemical shifts values before and after the addition of $\mathrm{Zn}\left(\mathrm{NO}_{3}\right)_{2}$ into the $\mathrm{CHH}$ system $\left(\Delta \delta=\left(\delta_{(\mathrm{CHH}-\mathrm{Zn})}-\delta_{(\mathrm{CHH})}\right)\right.$ was calculated in ppb and plotted as a function of amide, aromatic and aliphatic protons. 
Microfluidics experiments. Microfluidics experiments were performed as we previously reported. ${ }^{1}$ In a typical protocol, $\mathrm{CHH}-\mathrm{Zn}\left(\mathrm{NO}_{3}\right)_{2}$ crystalline powder was inserted into the device. Then, a flow of fresh solutions was injected at a rate of $4 \mu \mathrm{h}^{-1}$ using Cetoni GmbH neMESYS Syringe Pumps (Korbussen, Germany) and glass HAMILTON syringes, 1,725 TLL of $250 \mu$ l. The process was examined under an Eclipse Ti-E inverted microscope (Nikon, Japan), equipped with a Zyla 4.2+ sCMOS camera (Andor, UK), and images were captured at different time points.

Cyclic voltammetry. Electrochemical experiments were carried out using a CHI660A electrochemical workstation; Indium-tin-oxide (ITO) glass substrates $(10 \mathrm{~mm} \times 50 \mathrm{~mm} \times 0.7 \mathrm{~mm})$ served as a working electrode, $\mathrm{Ag} / \mathrm{AgCl}$ as the reference electrode, and a Pt wire as the counter electrode. CHH-Zn were dissolved in dry dimethylformamide (DMF) with 0.1 M tetrabutylammonium hexafluorophosphate $\left(\mathrm{TBAPF}_{6}\right)$ as the supporting electrolyte. All electrochemical measurements were performed in a nitrogen atmosphere.

Photoluminescence device fabrication and characterization. Commercially available InGaN chips were used at the bottom of the light emitting diode (LED) base. For preparation of the color conversion layer, the $\mathrm{CHH}-\mathrm{Zn}$ was blended into PVP at a mass ratio of 1: 70, and the resulting mixtures were vacuum-dried at $60{ }^{\circ} \mathrm{C}$ for $30 \mathrm{~min}$. The mixtures were applied on the InGaN chips and following curing at $80{ }^{\circ} \mathrm{C}$ for one hour, and the LEDs peptide phosphors were obtained.

Organic LED (OLED) device fabrication and characterization. ITO-coated glass substrates were cleaned ultrasonically in organic solvents (acetone and isopropyl alcohol), rinsed in deionized water, and then dried in an oven at $150{ }^{\circ} \mathrm{C}$ for $10 \mathrm{~min}$. The substrates were cleaned by a UV-ozone treatment to enrich the ITO surface with oxygen, thereby increasing its work function. The approximately $30 \mathrm{~nm}$ thick PEDOT: PSS hole injection layer was spin-coated at $3000 \mathrm{rpm}$ for $30 \mathrm{~s}$ on the ITO, followed by annealing in an oven at $150{ }^{\circ} \mathrm{C}$ for $15 \mathrm{~min}$. Subsequently, the emissive layer of CHH-Zn blended into PVK was spin-coated at $3000 \mathrm{rpm}$ for $35 \mathrm{~s}$ over the surface of the PEDOT:PSS film from the solution of NMP, followed by baking on a hot plate at $80^{\circ} \mathrm{C}$ for $15 \mathrm{~min}$ to form the active region of the peptide-derived bio-OLED. Finally, the substrates were transferred to a vacuum chamber and a $30 \mathrm{~nm}$ thick TPBI electron transport layer was thermally deposited with base pressure of $3 \times 10^{-4} \mathrm{~Pa}$. Next, a $20 \mathrm{~nm} \mathrm{Ca}$ and $100 \mathrm{~nm}$ thick Al cathode was deposited using a shadow mask $2 \mathrm{~mm}$ in width. The active area of the devices was thus $4 \mathrm{~mm}^{2}$. The thermal deposition 
rates for TPBI and $\mathrm{Ca} / \mathrm{Al}$ were 1,1 , and $3 \AA \mathrm{s}^{-1}$, respectively. The thickness of the films was measured using a Dektak XT (Bruker) surface profilometer and a spectroscopic ellipsometer (Suntech). The luminance-current-voltage (L-I-V) characteristics were measured using a computer-controlled Keithley 236 SMU and Keithley 200 multimeter coupled with a calibrated Si photodiode. Electroluminescence spectra were measured by an Ocean Optics 2000 spectrometer, which couples a linear charge-coupled device array detector ranging from 350 to $800 \mathrm{~nm}$.

Preparation of CHH-Zn+EPI. Fresh stock solutions of CHH were prepared by dissolving the peptide into $4 \%(\mathrm{v} / \mathrm{v}) \mathrm{DMSO} /$ isopropanol at a concentration of $5.48 \mathrm{mg} \mathrm{mL}^{-1}$. Subsequently, 0.25 mg EPI and $2.97 \mathrm{mg}$ of metal salt $\mathrm{Zn}\left(\mathrm{NO}_{3}\right)_{2}$ were added under vigorous sonication, followed by incubation in an $80{ }^{\circ} \mathrm{C}$ water bath for 1 hour and overnight at room temperature. The obtained suspension was then centrifuged at $15000 \mathrm{rpm}$ for $20 \mathrm{~min}$ and the precipitates were washed three times with Milli-Q water to remove any excess EPI and salts. To determine the loading capacity of EPI, the precipitated CHH-Zn+EPI nanoparticles were re-dissolved in DMSO and measured by UVVis absorption spectra with a range of known standard concentrations.

Release profile of CHH-Zn+EPI. CHH-Zn+EPI samples $(4 \mu \mathrm{g} / \mathrm{mL})$ and EPI $(0.63 \mu \mathrm{g} / \mathrm{mL})$ were individually placed into $3.5 \mathrm{kDa}$ dialysis tubes and dialyzed in $70 \mathrm{~mL}$ PBS buffer and acetate buffer at different $\mathrm{pH}$ values ( $\mathrm{pH} 7.4$ or 6.0). The dialysis was carried out by stirring inside an incubator shaker at $37{ }^{\circ} \mathrm{C}$ in the dark. Drug release was assumed to begin as soon as the dialysis chambers were placed into the buffer reservoirs. Aliquots $(100 \mu \mathrm{L})$ of the solutions in the release reservoirs were removed for characterization at various timepoints. The concentration of released EPI was determined by measuring the absorption at $500 \mathrm{~nm}$ using a calibration curve prepared under the same conditions.

Determination of cytotoxicity. $2 \times 10^{5}$ cells $/ \mathrm{mL}$ of HeLa cells were cultured in $96-$ well tissue microplates $\left(100 \mu \mathrm{L}\right.$ per well) and allowed to adhere overnight at $37{ }^{\circ} \mathrm{C}$. CHH-Zn+EPI was added in cell growth medium at concentrations of 1,2 , and $4 \mu \mathrm{g} / \mathrm{mL}$. Half of each plate was seeded with cells, while the other half was used as a blank control. Medium with no CHH-Zn+EPI served as a negative control. After overnight incubation (12 hours) at $37^{\circ} \mathrm{C}$, cell viability was evaluated using 3-(4,5-dimethylthiazolyl-2)-2, 5-diphenyltetrazolium bromide MTT cell proliferation assay according to the manufacturer's instructions. Briefly, after overnight incubation at $37^{\circ} \mathrm{C}$ with the 
CHH-Zn+EPI, $10 \mu \mathrm{L}$ of $5 \mathrm{mg} / \mathrm{mL}$ MTT reagent dissolved in PBS was added to each of the 96 wells, followed by a $4 \mathrm{~h}$ incubation at $37^{\circ} \mathrm{C}$. Next, $100 \mu \mathrm{L}$ extraction buffer $(50 \% \mathrm{DMF}, 20 \%$ SDS in Milli-Q water) was added to the wells, followed by 30 min incubation at $37^{\circ} \mathrm{C}$ in the dark. Finally, color intensity was measured using an ELISA plate reader at $570 \mathrm{~nm}$ and background subtraction at $680 \mathrm{~nm}$.

Live cells imaging using confocal microscopy (CLSM). HeLa cells were grown to $70-80 \%$ confluence in glass bottom cell culture dishes. Then, the cells were cultured with media containing the $\mathrm{CHH}-\mathrm{Zn}+\mathrm{EPI}$ at a concentration of $4 \mu \mathrm{g} / \mathrm{mL}$ for different durations. Next, the cells were stained using a DRAQ5 ${ }^{\mathrm{TM}}$ dye diluted 1:1000 in PBS for 15 minutes at room temperature in the dark to allow staining of the nuclei. The cells were then washed twice with PBS. Imaging was performed using SP8 inverted confocal microscope (Leica Microsystems, Wetzlar, Germany). Excitation and emission ranges: $\lambda_{\mathrm{ex}}=405 \mathrm{~nm}, \lambda_{\mathrm{em}}=420-500 \mathrm{~nm}$; EPI, $\lambda_{\mathrm{ex}}=543 \mathrm{~nm}, \lambda_{\mathrm{em}}=550-750 \mathrm{~nm}$; DRAQ5, $\lambda_{\mathrm{ex}}=633 \mathrm{~nm}, \lambda_{\mathrm{em}}=750-780 \mathrm{~nm}$.

FLIM analysis of cultured cells. HeLa cells seeded in dishes were treated with $\mathrm{CHH}-\mathrm{Zn}+\mathrm{EPI}$ at a concentration of $4 \mu \mathrm{g} \mathrm{mL}^{-1}$ for $30 \mathrm{~min}, 76 \mathrm{~min}, 125 \mathrm{~min}, 194 \mathrm{~min}, 270 \mathrm{~min}$, and $420 \mathrm{~min}$, followed by washing with PBS. The time-resolved fluorescence signal was acquired using an LSM 7 MP two-photon microscope (Carl Zeiss, Weimar, Germany) coupled to the Becker and Hickl (BH) simple-Tau-152 system. Images were acquired through a Zeiss $20 \mathrm{X} / 1 \mathrm{NA}$ water-immersion objective. A Zeiss dichroic mirror (T690) was used to separate the excitation and the emission light. An additional barrier filter was used to block emission light above $690 \mathrm{~nm}$. Emission light was separated by a dichroic mirror $(555 \mathrm{~nm})$ and the two fluorescent lights were filtered by two bandpass filters (500-550 nm and 590-650 nm). Pseudocolored lifetime images were generated by assigning a colour to the value of average fluorescence lifetime $\tau_{\mathrm{m}}$ at each pixel. Emission light was collected via a hybrid GaAsP detector (HPM-100-40, BH, Berlin, Germany) with a Cherry bandpass filter.

Phasor analysis of FLIM data. The phasor-FLIM analysis was performed using the SPCImage 6.4 software. The fluorescence signal collected from each pixel of the image was transformed into Fourier space and a phasor map was constructed. From the FLIM measurements, the sine (S) and cosine $(G)$ Fourier components of the lifetime decay were calculated for every pixel of the image, yielding the two phasor coordinates G and S, calculated using Supplementary equations Eq. S(1) 
and Eq. S(2):

$$
\begin{aligned}
s_{i, j(\omega)}=\frac{\int_{0}^{\infty} \mathrm{I}(\mathrm{t}) \sin (n \omega t) d t}{\int_{0}^{\infty} \mathrm{I}(\mathrm{t}) d t} & \text { Eq. } \mathrm{S} 1 \\
g_{i, j(\omega)}=\frac{\int_{0}^{\infty} \mathrm{I}(\mathrm{t}) \cos (n \omega t) d t}{\int_{0}^{\infty} \mathrm{I}(\mathrm{t}) d t} & \text { Eq. } \mathrm{S} 2
\end{aligned}
$$

where the indices $i$ and $j$ represent the pixel of the image and $I(t)$ represents the photon counts of the time bin, $t$, of the lifetime decay histogram of the corresponding pixel. $\omega=2 \pi f$, where $f$ is the laser repetition frequency (i.e., $80 \mathrm{MHz}$ in our experiments) and $\mathrm{n}$ is the harmonic frequency. Analysis of the phasor distribution was performed by cluster identification. In general, every possible lifetime can be mapped onto this universal representation of the decay (phasor plot), and multiple species are added vectorially. To obtain a single apparent lifetime for each sample, a cluster of pixel values were detected in specific regions of the phasor plot, and the average fluorescence lifetime $\tau_{\mathrm{m}}$ was calculated from all pixels above a threshold of $\sim 300$ photons.

\section{Computational analysis}

Modeling of the investigated systems. Three systems, $\mathrm{CHH}, \mathrm{Zn}(\mathrm{II})$, and $\mathrm{NO}_{3}{ }^{-}$in isopropanol, $\mathrm{CHH}$ and $\mathrm{Zn}(\mathrm{II})$ in isopropanol, and $\mathrm{CHH}, \mathrm{Zn}(\mathrm{II}), \mathrm{NO}_{3}{ }^{-}$and EPI in isopropanol, were modeled. In the former two systems, $64 \mathrm{CHH}$ molecules were introduced into a $100 \AA^{3}$ isopropanol box, modeling a CHH concentration of $36 \mathrm{mg} / \mathrm{mL}$. The concentration was higher than used in the experimental studies, aiming to accelerate the self-assembly within the frame of the simulations, and facilitate the computational investigation of the systems. ${ }^{2-3}$ In both systems, CHH dipeptides were embedded in a $60 \AA^{3}$ grid within the isopropanol box, with an equal spacing of $\sim 15 \AA$ and random configurations $\&$ orientations. The random configurations were based on an extra-short simulation of $\mathrm{CHH}$ at infinite dilution. In both systems, the initial placement of $\mathrm{CHH}$ in the grid was performed five times in a random fashion. Subsequently, $\mathrm{Zn}$ (II) ions were additionally placed in a grid such that they were in the vicinity of the $\mathrm{CHH}$ dipeptides in the five different initial arrangements produced above. Nevertheless, the placement was performed without any intentional interactions between the ions and the dipeptides. As a result, five different initial arrangements of $\mathrm{CHH}$ and $\mathrm{Zn}(\mathrm{II})$ in isopropanol were produced, which were later used as the different simulated initial starting structures to enhance the statistical sampling. In the $\mathrm{CHH}+\mathrm{Zn}$ (II) system, chloride ions were subsequently introduced to neutralize the overall charge of the simulation system through 2000 steps of Monte Carlo 
simulations. ${ }^{4}$ In the $\mathrm{CHH}+\mathrm{Zn}(\mathrm{II})+\mathrm{NO}_{3}{ }^{-}$system, nitrate ions were introduced to neutralize the overall charge of the simulation system through 2000 steps of Monte Carlo simulations. ${ }^{4}$

In the latter system comprising $\mathrm{CHH}, \mathrm{Zn}(\mathrm{II}), \mathrm{NO}_{3}{ }^{-}$, and $\mathrm{EPI}$ in isopropanol, $32 \mathrm{CHH}$ and $8 \mathrm{EPI}$ molecules were introduced into a $100 \AA^{3}$ isopropanol box, modeling a CHH concentration of 18 $\mathrm{mg} / \mathrm{mL}$ and an EPI concentration of $2.25 \mathrm{mg} / \mathrm{mL}$. The concentration was higher than used in the experimental studies, aiming to accelerate the self-assembly within the frame of the simulations, and facilitate the computational investigation of the system. ${ }^{2-3}$ Subsequently, $\mathrm{Zn}(\mathrm{II})$ ions were additionally placed in a grid such that they were in the vicinity of the $\mathrm{CHH}$ in the five different initial arrangements produced above. Nevertheless, the placement was performed without any intentional interactions between the ions and the dipeptides. Nitrate ions were additionally introduced to neutralize the overall charge of the simulation system through 2000 steps of Monte Carlo simulations. $^{4}$

Molecular dynamics (MD) simulations. MD simulations of the three systems modeled and described above, $\mathrm{CHH}, \mathrm{Zn}(\mathrm{II})$, and $\mathrm{NO}_{3}{ }^{-}$in isopropanol, $\mathrm{CHH}$ and $\mathrm{Zn}(\mathrm{II})$ in isopropanol, and $\mathrm{CHH}$, $\mathrm{Zn}(\mathrm{II}), \mathrm{NO}_{3}{ }^{-}$, and EPI in isopropanol, were performed. All systems were simulated in five-replicates for a duration of $100 \mathrm{~ns}$ per simulation run. Prior to the execution of MD simulations, energy minimization was performed. $\mathrm{CHH}$ dipeptides were first fixed to their initial conformations, and 50 steps of steepest descent followed by 50 steps of Adopted Basis Newton-Raphson energy minimization were performed to alleviate any clashes, primarily between the $\mathrm{CHH}$ molecules and their surrounding environment. Subsequently, the heavy atoms of $\mathrm{CHH}$ were constrained with 0.1 kcal $\mathrm{mol}^{-1} \AA^{-2}$ harmonic constraints at their initial positions for additional 100 steps of steepest descent, followed by 100 steps of Adopted Basis Newton-Raphson energy minimization and $1 \mathrm{~ns}$ equilibration of the surrounding environment.

Finally, five MD simulation production runs, $100 \mathrm{~ns}$ each, were performed for each of the three systems. Five replicate MD simulation runs were preferred over a single run for increased sampling and to ensure that the formation of clusters was observed within multiple simulations and were not an artifact of the molecules being trapped in a local energetic minimum in a given simulation. During the production runs, no constraints were imposed to the system. All energy minimization and MD simulations were performed using the CHARMM36 force field ${ }^{5}$ and periodic boundary 
conditions in CHARMM. ${ }^{6}$ The temperature of the simulation systems was maintained at $300 \mathrm{~K}$ using the dual Nosé-Hoover thermostat, ${ }^{7}$ and the pressure was maintained at $1.0 \mathrm{~atm}$ using the Andersen-Hoover barostat. ${ }^{8}$ The bond lengths of covalently bonded hydrogens were constrained using the SHAKE algorithm. ${ }^{9}$ Upon completion of the MD simulations, the five independent simulation trajectories were merged, resulting in a total of 2500 snapshots analyzed per system.

Structural analysis of MD simulations. Self-assembly of CHH dipeptides into clusters from their initial positioning in random orientations was observed in all systems. In-house FORTRAN programs (available upon request) were used to analyze the $\mathrm{CHH} / \mathrm{CHH}, \mathrm{Zn}(\mathrm{II}) / \mathrm{CHH}, \mathrm{NO}_{3}{ }^{-} / \mathrm{CHH}$, Epirubicin/CHH, $\mathrm{Zn}(\mathrm{II}) / \mathrm{NO}_{3}{ }^{-}, \mathrm{NO}_{3}{ }^{-} /$Epirubicin, and $\mathrm{Zn}(\mathrm{II}) /$ Epirubicin interactions within the clusters, which can potentially correspond to the structures formed in the first instances of association. Pairs of entities (CHH, Epirubicin, $\mathrm{Zn}(\mathrm{II})$, or $\mathrm{NO}_{3}^{-}$) were considered to be interacting if any of their atoms were within $3.5 \AA$ of each other. A number of $n$ entities (CHH, Epirubicin, $\mathrm{Zn}$ (II), or $\mathrm{NO}_{3}^{-}$) were defined to form a cluster when each dipeptide was in the vicinity of at least another one based on the $3.5 \AA$ distance criterion defined above.

Subsequently, for each cluster identified within the simulations, we investigated the geometric properties. For the simulations comprising $\mathrm{CHH}, \mathrm{Zn}(\mathrm{II})$ and $\mathrm{NO}_{3}{ }^{-}$in isopropanol and $\mathrm{CHH}, \mathrm{Zn}(\mathrm{II})$, $\mathrm{NO}_{3}^{-}$, and EPI in isopropanol, the percent solvent exposure (solvent accessible surface area (SASA)/molecular surface area) was calculated for each entity within a given cluster (CHH, $\mathrm{Zn}$ (II), or $\mathrm{NO}_{3}{ }^{-} / \mathrm{CHH}, \mathrm{Zn}(\mathrm{II}), \mathrm{NO}_{3}{ }^{-}$, or EPI) to determine the existence of exterior and interior layers and their composition within the observed clusters. All SASA and molecular surface area calculations were performed using the GEPOL algorithm in Wordom. ${ }^{10-11}$ The probe radius for isopropanol was set to $2.2 \AA .{ }^{12-13}$ Subsequently, we calculated the running average percent exposure of each entity within a cluster beginning from the most buried entity (lowest percent exposure) and moving outwards. Entities with a running average percent exposure less than or equal to $45 \%$ were considered to be in the interior layer of the clusters, whereas entities with a running average percent exposure greater than $45 \%$ were considered to be at the exterior layer of the clusters (Figure $3 \mathrm{c}$ in the main text, Figure S19a). The $45 \%$ exposure cutoff was selected based on visual inspection of the simulated clusters. Upon delineation of entities within the interior and exterior layers of a given cluster, we calculated the percent population of $\mathrm{CHH}, \mathrm{Zn}(\mathrm{II}), \mathrm{NO}_{3}{ }^{-}$or $\mathrm{CHH}, \mathrm{Zn}(\mathrm{II}), \mathrm{NO}_{3}{ }^{-}$, and EPI 
within the interior and exterior layers of the cluster, independently.

For the simulations comprising $\mathrm{CHH}, \mathrm{Zn}(\mathrm{II})$, and $\mathrm{NO}_{3}{ }^{-}$in isopropanol and $\mathrm{CHH}, \mathrm{Zn}(\mathrm{II})$ in isopropanol, we calculated the radius of gyration of $\mathrm{Zn}(\mathrm{II})$ within each observed cluster to determine and compare the compactness of the $\mathrm{Zn}$ (II) in the two systems (Figure 3a,b). For the simulations comprising $\mathrm{CHH}, \mathrm{Zn}(\mathrm{II}), \mathrm{NO}_{3}{ }^{-}$, and EPI in isopropanol, we calculated the radius of gyration of $\mathrm{CHH}$ and EPI, independently, within each cluster to determine if $\mathrm{CHH}$ were encapsulating EPI (Figure $\mathrm{S} 19 \mathrm{~b})$. The radius of gyration was calculated using the following equation in Wordom: ${ }^{10-11}$

$$
R_{g}=\sqrt{\frac{1}{N} \sum_{k=1}^{N}\left(r_{k}-\bar{r}\right)^{2}}
$$

In the above equation, the radius of gyration $\left(R_{g}\right)$ is the square root of the average deviation of $N$ atoms $\left(r_{k}\right)$ from the geometric center $(\bar{r})$. For the simulations comprising $\mathrm{CHH}, \mathrm{Zn}(\mathrm{II})$, and $\mathrm{NO}_{3}{ }^{-}$in isopropanol and $\mathrm{CHH}$ and $\mathrm{Zn}(\mathrm{II})$ in isopropanol, the radius of gyration calculations were performed with only the $\mathrm{Zn}(\mathrm{II})$ ions within each cluster considered and all other atoms omitted, aiming to compare the compactness of the $\mathrm{Zn}(\mathrm{II})$ within clusters formed in the presence of $\mathrm{NO}_{3}{ }^{-}$to those formed in the absence of $\mathrm{NO}_{3}{ }^{-}$. Larger values of gyration radius indicate lower compactness, or lower packing density and concentration of $\mathrm{Zn}(\mathrm{II})$ in the clusters, while lower gyration values indicate higher compactness, or higher density and concentration of $\mathrm{Zn}(\mathrm{II})$ in the clusters. For the simulations comprising $\mathrm{CHH}, \mathrm{Zn}^{2+}, \mathrm{NO}_{3}{ }^{-}$, and $\mathrm{EPI}$ in isopropanol, the radius of gyration calculations were performed with only $\mathrm{CHH}$ or Epirubicin, independently, within each cluster considered with all other atoms omitted. The radius of gyration of Epirubicin within a given cluster was subsequently subtracted from the radius of gyration of $\mathrm{CHH}$ of the same cluster. Thus, positive value indicates a larger radius of gyration in the $\mathrm{CHH}$ layer than in the EPI layer within a given cluster.

For the simulations comprising $\mathrm{CHH}, \mathrm{Zn}(\mathrm{II})$, and $\mathrm{NO}_{3}{ }^{-}$in isopropanol and $\mathrm{CHH}, \mathrm{Zn}(\mathrm{II}), \mathrm{NO}_{3}{ }^{-}$, and Epirubicin in isopropanol, we additionally investigated the time evolution properties of their individual entities for clusters made up of the entities $\left(\mathrm{CHH}, \mathrm{Zn}(\mathrm{II}), \mathrm{NO}_{3}{ }^{-}\right.$or Epirubicin) that eventually form clusters containing at least $10 \mathrm{CHH}$ in the former set of simulations and for clusters made up of the entities (CHH, $\mathrm{Zn}(\mathrm{II})$, or Epirubicin) that eventually form clusters containing at least 10 molecules with a composition ranging from $30 \% \mathrm{CHH}$ and $70 \%$ EPI to $70 \% \mathrm{CHH}$ and $30 \%$ Epirubicin in the latter set of simulations. The analysis was focused on clusters containing at least 
$10 \mathrm{CHH}$ or 10 molecules as they are more complex than clusters containing fewer molecules and were also sufficiently populated for a statistical analysis.

Energetic analysis of MD simulations. To investigate the mechanism and driving forces leading to association and stabilization of the clusters formed by $\mathrm{CHH}, \mathrm{Zn}(\mathrm{II})$, and $\mathrm{NO}_{3}{ }^{-}$or $\mathrm{CHH}, \mathrm{Zn}(\mathrm{II})$, $\mathrm{NO}_{3}{ }^{-}$and Epirubicin, we performed association free energy calculations using the MM-GBSA approximation $^{14-15}$ (Figure S18). For a given cluster, we calculated the association free energies such that the cluster was isolated to predict possible pathways of formation. The association free energy calculations were performed over an ensemble of clusters to ensure statistical significance. In the association free energy calculations, the Generalized Born with a simple Switching (GBSW) implicit-solvent mode ${ }^{16}$ was used to account for the solvent. For the simulation system comprising $\mathrm{CHH}, \mathrm{Zn}(\mathrm{II})$, and $\mathrm{NO}_{3}{ }^{-}$, the solvent dielectric constant was set to $18.44^{17}$ to account for the dielectric environment of isopropanol. For both simulation systems, non-polar solvation effects were omitted from the calculations reported in the main text. Such contributions can be calculated through a surface tension coefficient multiplying the solvent accessible surface area, and the surface tension coefficient can be obtained by fitting the experimental hydration energies of saturated linear hydrocarbons. ${ }^{18}$ However, the reported values of the surface tension coefficient vary by over an order of magnitude ${ }^{19-20}$ depending on the definition of solute surface area, the training set, and the underlying force fields used to describe the solute molecule. ${ }^{21-23}$ As the simulation systems are solvated in isopropanol rather than in water, and thus comprehensive studies of the appropriate surface tension coefficient in balance with the GBSW-implicit solvent used are lacking, the nonpolar solvation effects were neglected to avoid any bias or inaccuracy driven by an arbitrarily chosen value. Nevertheless, additional calculations were performed using the surface tension coefficient's default value for GBSW, which corresponds to $0.03 \mathrm{kcal} \mathrm{mol}^{-1} \AA^{-2}$, appropriate for systems solvated in water, ${ }^{16}$ and the overall trends remained the same as provided in the main text and analytically presented below (data not shown).

Clusters of CHH-Zn( $\left(\mathrm{NO}_{3}\right)_{2}$ or $\mathrm{CHH}-\mathrm{Zn}\left(\mathrm{NO}_{3}\right)_{2}$ and Epirubicin were collected from the simulations, and free energy calculations were performed to elucidate the possible pathways of formation. For each cluster, an energy calculation was performed, with the energy normalized by the number of $\mathrm{CHH}$ dipeptides in the cluster. A series of association free energy calculations was 
performed, corresponding to the total energy of the formed clusters minus the sum of the individual energies of the interior nucleus and the exterior surface of the clusters in different pathways. The free energy to associate the clusters was calculated, (i) by assuming that the individual entities forming the cluster are initially not interacting, completely immersed in pure isopropanol, and spontaneously self-assemble into a cluster (Figure S18a,b, blue), (ii) by assuming that the interior nucleus and the exterior surface assemblies are individually preformed, initially not interacting and completely immersed in pure isopropanol, and subsequently aggregate to form a cluster (Figure S18a,b, black), (iii) by assuming that the exterior surface assembly is formed first in pure isopropanol and individual entities forming the interior nucleus are initially not interacting neither with the preformed exterior surface nor with each other and are completely immersed in pure isopropanol, and subsequently aggregate on the preformed exterior surface to form a cluster (Figure S18a,b, red), and (iv) by assuming that the interior nucleus assembly is formed first in pure isopropanol and individual entities of the exterior surface are initially not interacting neither with the preformed interior surface nor with each other and are completely immersed in pure isopropanol, and subsequently aggregate and wrap around the preformed nucleus to form a cluster (Figure S18a,b, green). We considered the most energetically favored pathway to be the mechanism by which the interior nucleus assembles first followed by individual exterior components wrapping the interior nucleus to form the clusters. The resulting pathway was validated using structural analysis (described above) calculating the properties of the formed clusters as a function of simulation time (Figure S19).

We aimed to obtain additional insights on the step-wise pathways that could lead to the formation of clusters by $\mathrm{CHH}-\mathrm{Zn}\left(\mathrm{NO}_{3}\right)_{2}+$ Epirubicin, with emphasis on the green encircled pathway of Figure S18b. One potential two-step pathway assumes first that the individual entities forming the cluster are initially not interacting, completely immersed in pure isopropanol, and Epirubucin molecules spontaneously form a cluster, completely immersed in pure isopropanol, while the rest of the entities are still immersed in pure isopropanol and neither interact with each other nor with Epirubicin molecules (Figure S18ci), and second that the remaining individual entities wrap around the preformed nucleus to form a cluster (Figure S18b, green and S18cii). An alternative, yet artificial pathway assumes first that $\mathrm{Zn}(\mathrm{II})$ would be artificially surrounded by the lower dielectric 
environment provided by the $\mathrm{CHH}$ within the final formed cluster, while the rest the entities are still immersed in pure isopropanol and neither interact with each other, and the epirubicin molecules form a cluster within the lower dielectric environment (Figure S18ciii), and second that the remaining individual entities wrap around the preformed nucleus to form a cluster (Figure S18b, green and S18civ). This artificial pathway was conducted to shed light into the role of the CHH-Zn environment in the initial clustering of Epirubucin molecules, and can be considered an overestimate of the absolute association free energy (or an underestimate of the actual association free energy).

According to these calculations, the free energy to associate the interior cluster assuming that all assembling counterparts are completely immersed in pure isopropanol prior to association is unfavorable $(7.6 \pm 2.3 \mathrm{kcal} / \mathrm{mol}$-molecule $)$ while the free energy to associate the interior cluster assuming that $\mathrm{Zn}(\mathrm{II})$ is surrounded by the lower dielectric environment provided by $\mathrm{CHH}$ within the final formed cluster is favorable $(-9.3 \pm 2.1 \mathrm{kcal} / \mathrm{mol}$-molecule) (Figure S18d). Additionally, the free energy to associate the cluster assuming that the interior cluster is preformed within the lower dielectric environment provided by the $\mathrm{CHH}$ within the final formed cluster is also favorable (Figure S18d). Considering the values obtained for the first pathway and the second artificial pathway, we suggest that the actual mechanism could be considered a combination of the two and a gradual transition from pure isopropanol toward the dielectric environment of $\mathrm{CHH}$ (Figure S18c, encircled in grey dotted lines), at which the association of the interior cluster is facilitated by the initial transfer of $\mathrm{Zn}(\mathrm{II})$ from an isopropanol to a peptide-like environment or "environment-switching” mechanism, ${ }^{24}$ enabling Epirubicin molecules to be self-encapsulated and further facilitating the eventual assembly of individual $\mathrm{CHH}$ and $\mathrm{NO}_{3}{ }^{-}$exteriorly wrapping around the preformed nucleus (Figure S18c, in grey dotted lines). 


\section{Supplementary Figures}
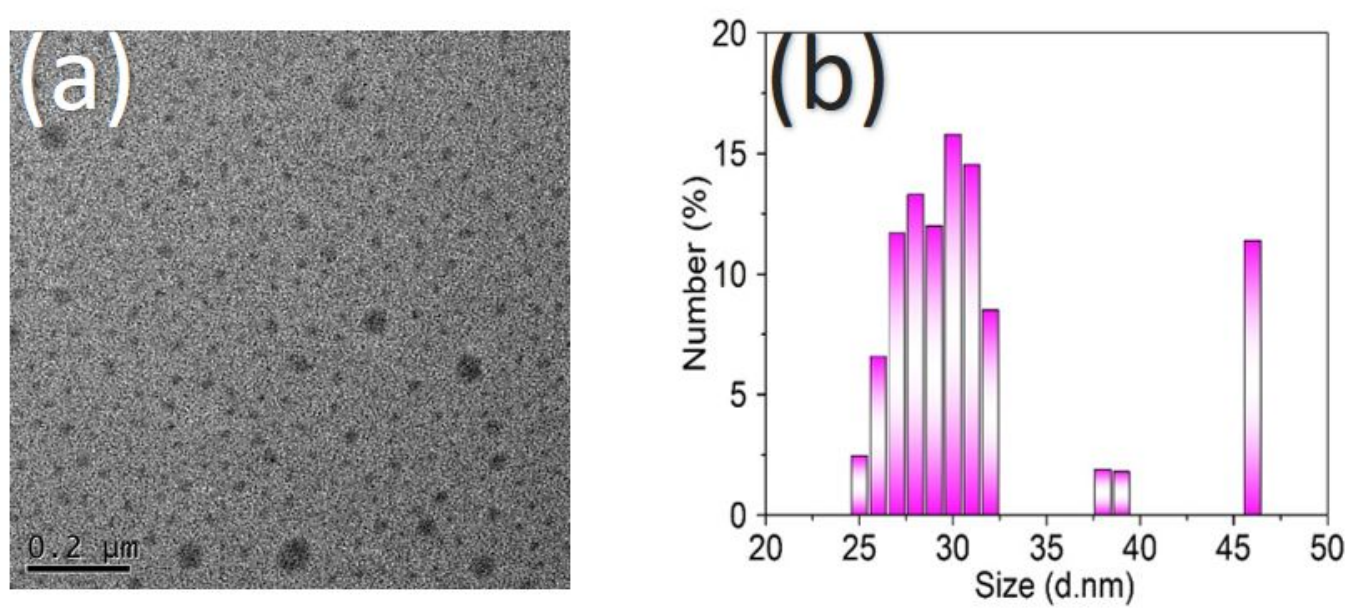

Supplementary Figure S1. (a) CHH-Zn TEM image. (b) Size distribution histogram of CHH-Zn counted from the TEM image. 


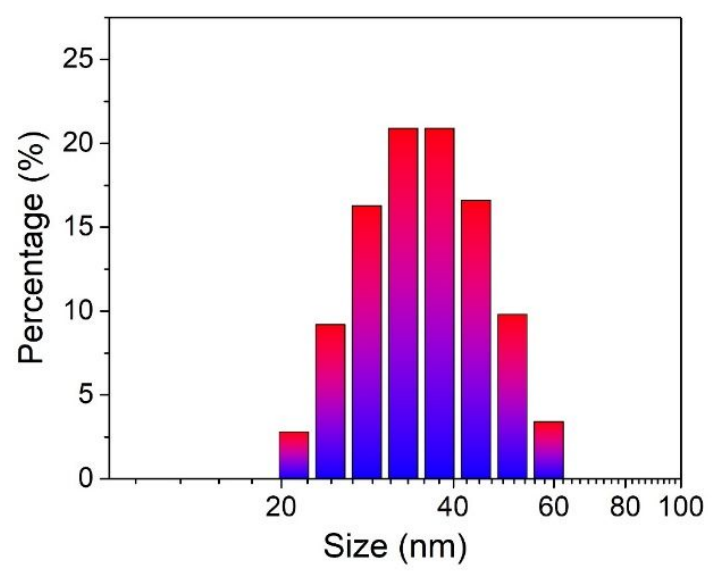

Supplementary Figure S2. Dynamic light-scattering measurement profiles of CHH-Zn. When the CHH dipeptide and $\mathrm{Zn}\left(\mathrm{NO}_{3}\right)_{2}$ were self-assembled, the predominant sizes were around $30 \sim 40 \mathrm{~nm}$, in accordance with the results of TEM and AFM analyses. 


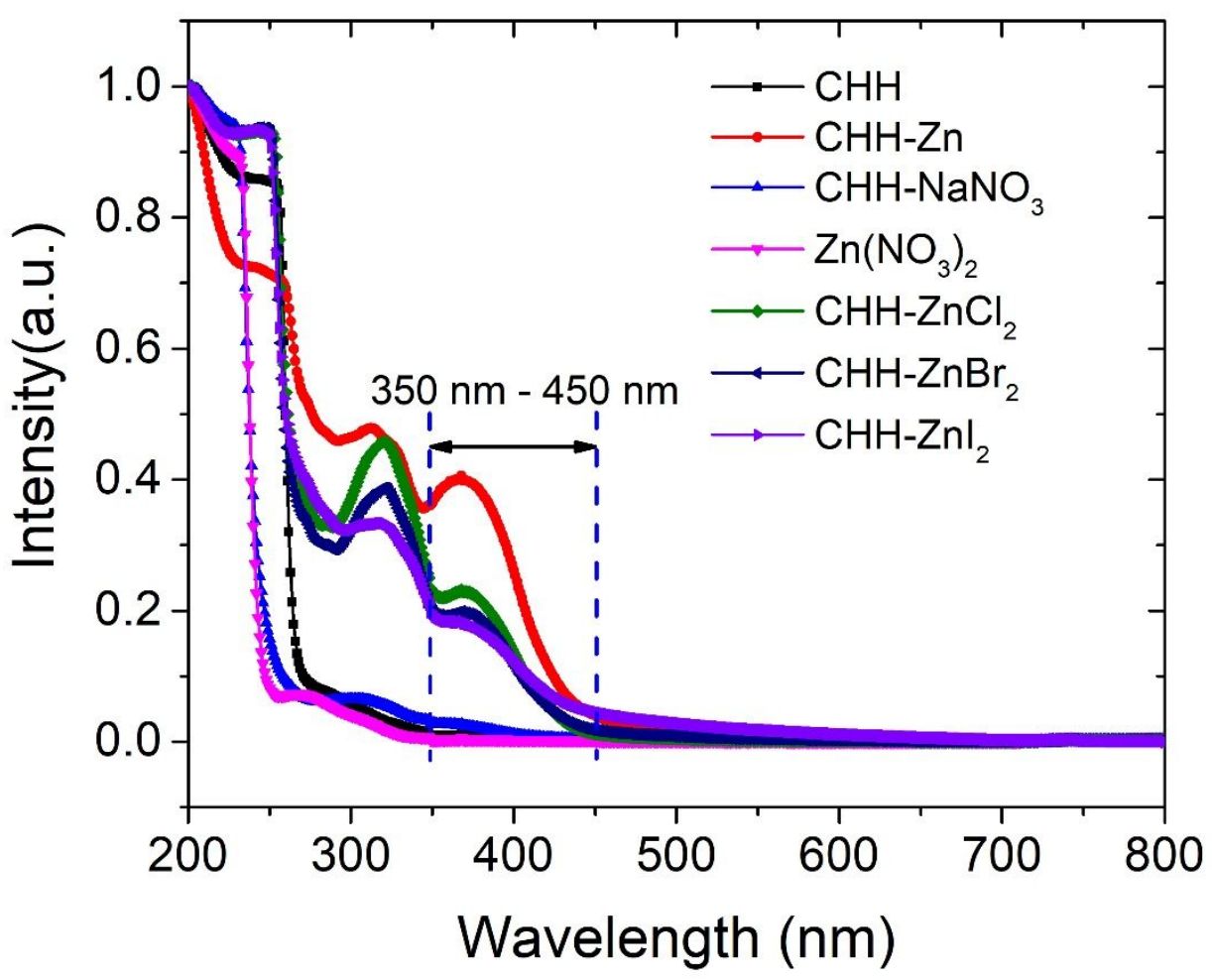

Supplementary Figure S3. Normalized UV-vis spectra of CHH-Zn, CHH- $\mathrm{ZnCl}_{2}, \mathrm{CHH}-\mathrm{ZnBr}_{2}$, CHH-ZnI $2, \mathrm{CHH}-\mathrm{NaNO}_{3}, \mathrm{CHH}$ and $\mathrm{Zn}\left(\mathrm{NO}_{3}\right)_{2}$. All $\mathrm{Zn}(\mathrm{II})$-coordinated assembly systems displayed similar absorption spectra. 

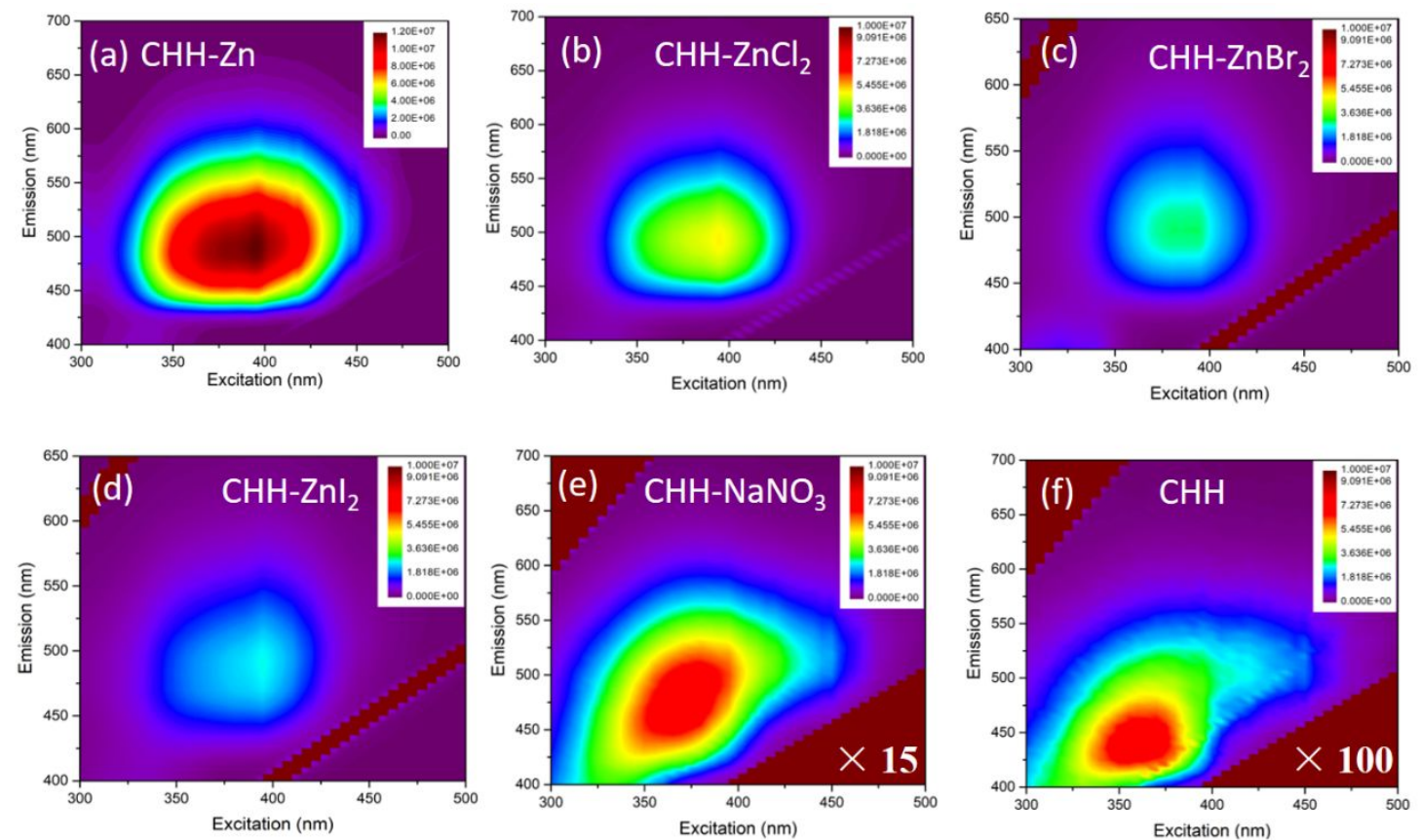

Supplementary Figure S4. Excitation-emission matrix contour profiles of (a) CHH-Zn, (b) CHH$\mathrm{ZnCl}_{2}$, (c) $\mathrm{CHH}-\mathrm{ZnBr}_{2}$, (d) $\mathrm{CHH}-\mathrm{ZnI}_{2}$, (e) $\mathrm{CHH}-\mathrm{NaNO}_{3}$ (intensity*15), and (e) $\mathrm{CHH}$ (intensity*100). All Zn(II)-coordinated assembly systems displayed strong fluorescence signal and similar excitation-emission matrix contour profiles. The sodium nitrate-related self-assembly system and $\mathrm{CHH}$ showed similar fluorescence profiles with an emission wavelength of $450 \mathrm{~nm}$. 


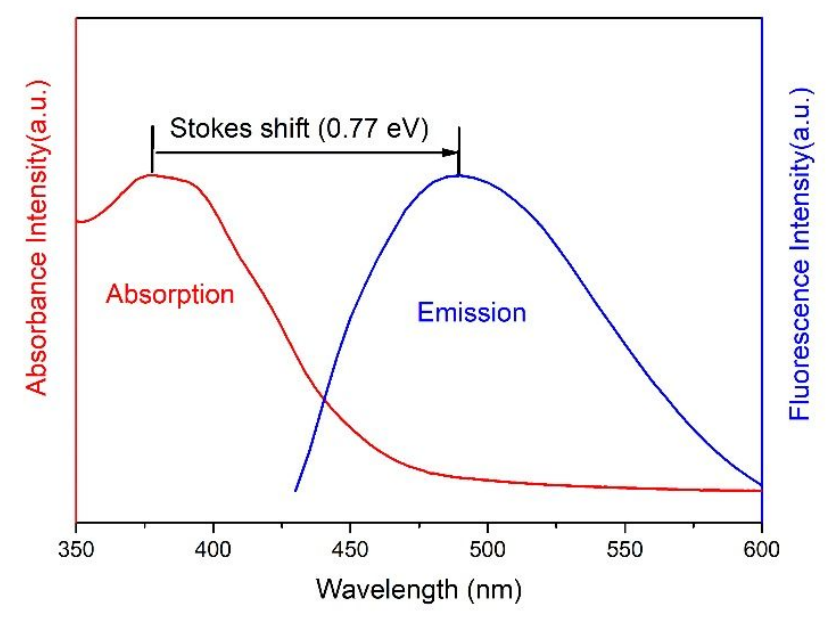

Supplementary Figure S5. Normalized absorbance spectra and normalized emission spectra of CHH-Zn. 


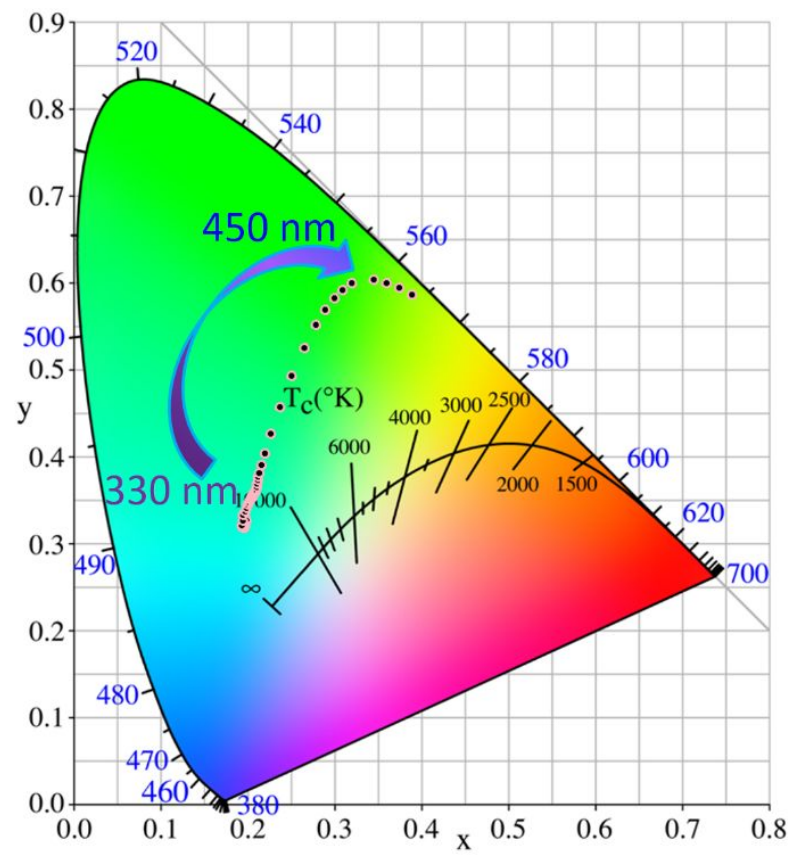

Supplementary Figure S6. Trajectory of tunable fluorescence emission colors recorded upon changing the excitation wavelength of CHH-Zn from 330 to $450 \mathrm{~nm}$ in the CIE coordinate diagram. 

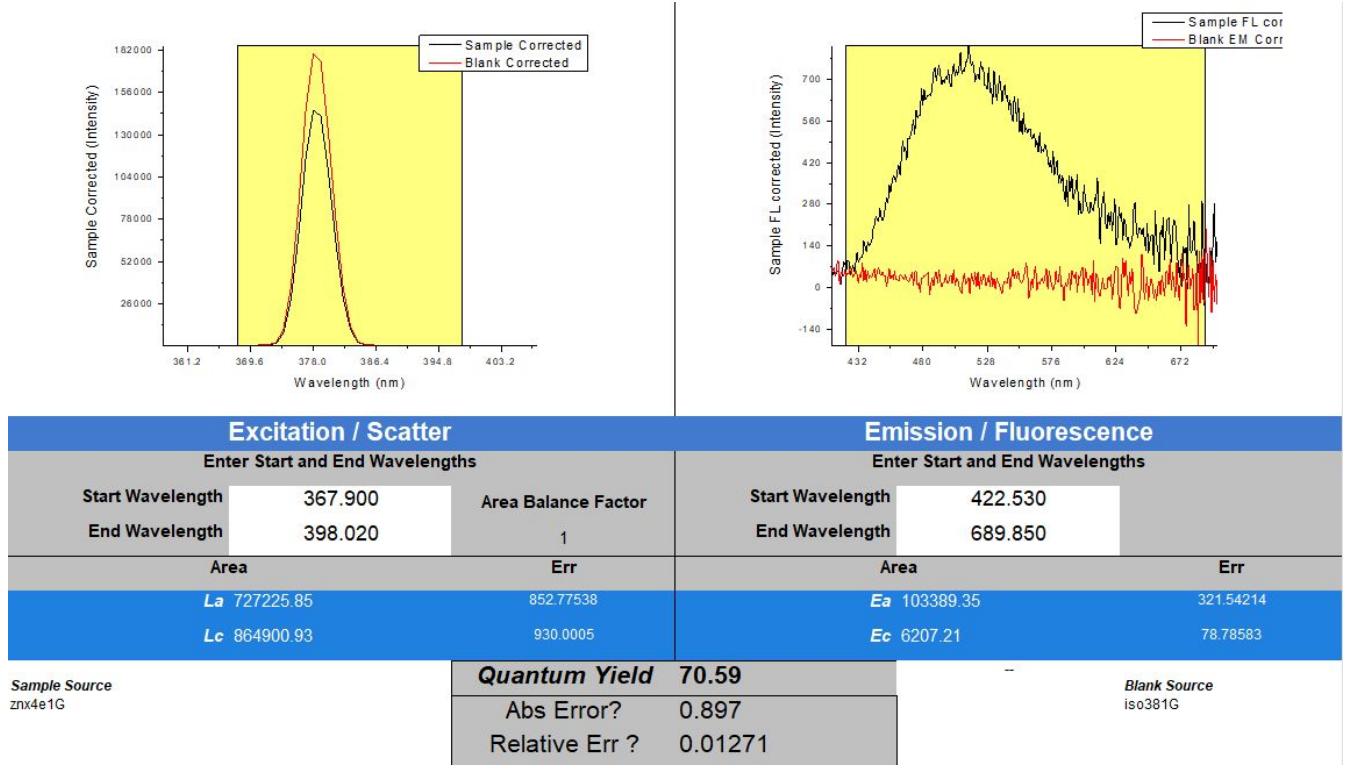

Supplementary Figure S7. Quantum yield of CHH-Zn measured under excitation of $380 \mathrm{~nm}$ at room temperature. 


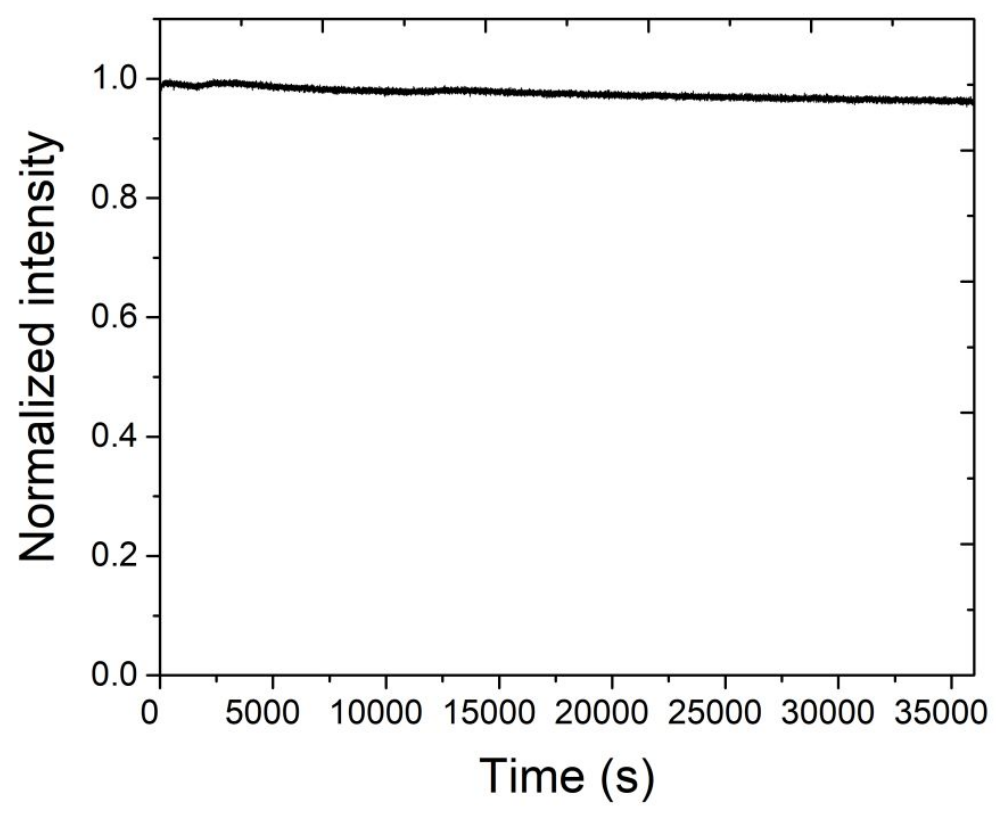

Supplementary Figure S8. Photostability evaluation of CHH-Zn under excitation of $380 \mathrm{~nm}$ at room temperature. The fluorescence intensity of $\mathrm{CHH}-\mathrm{Zn}$ remained stable after continuous irradiation for 36000 seconds. 


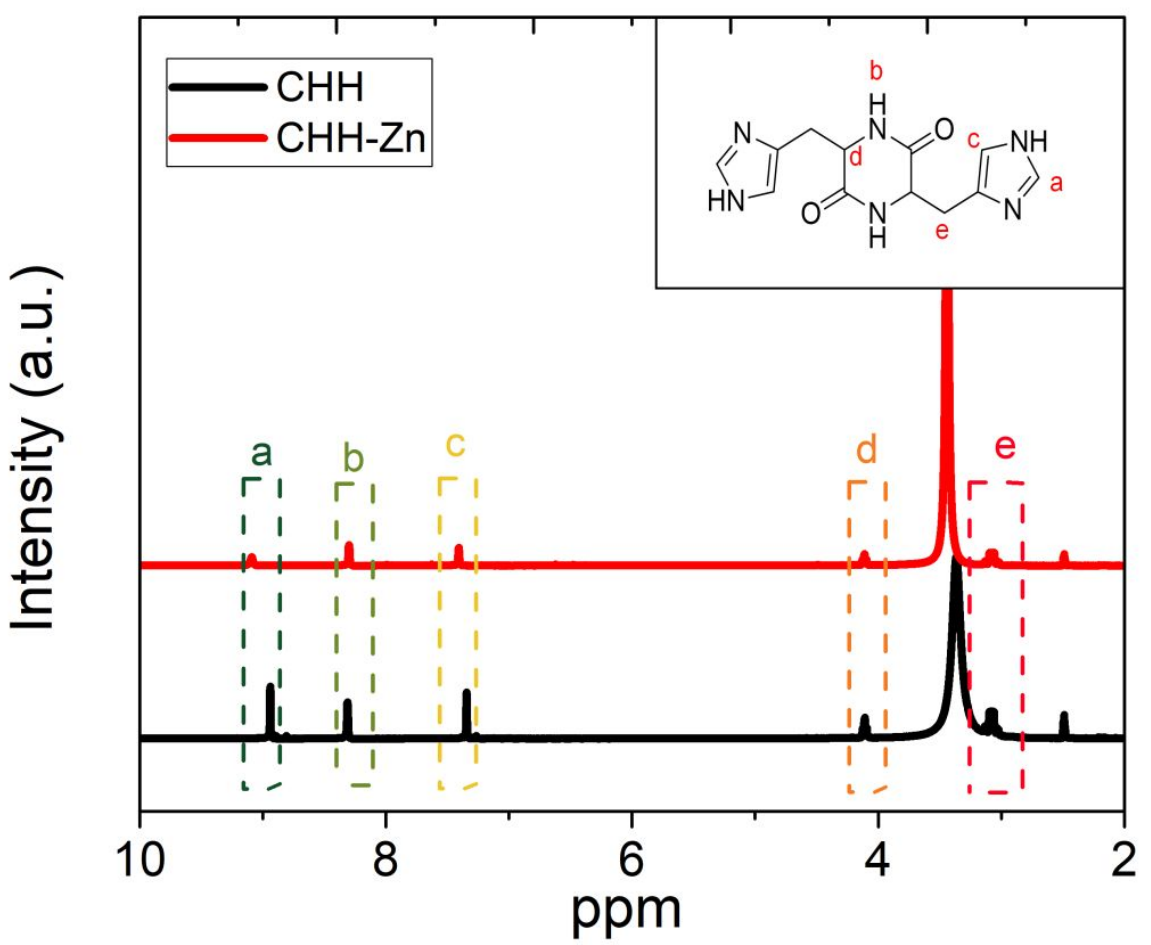

Supplementary Figure S9. Chemical shifts of CHH hydrogen atoms upon coordination with $\mathrm{Zn}\left(\mathrm{NO}_{3}\right)_{2}$, compared to the peptide alone. 
(a) 3

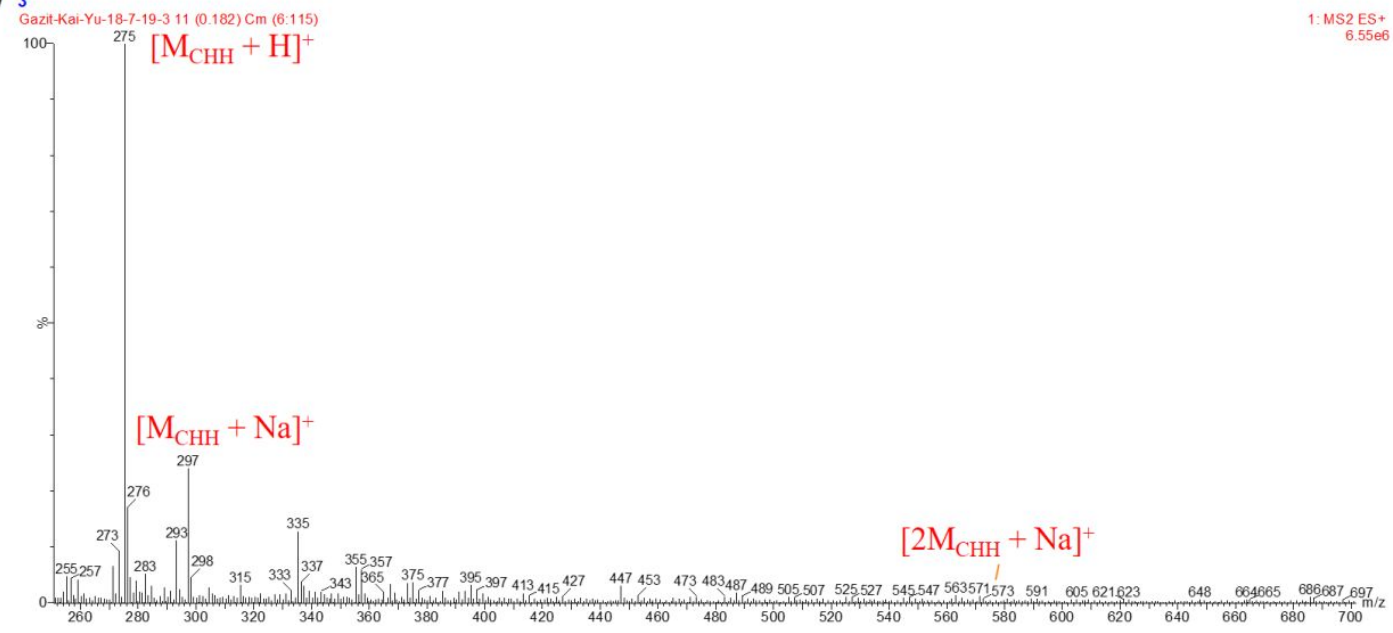

(b)

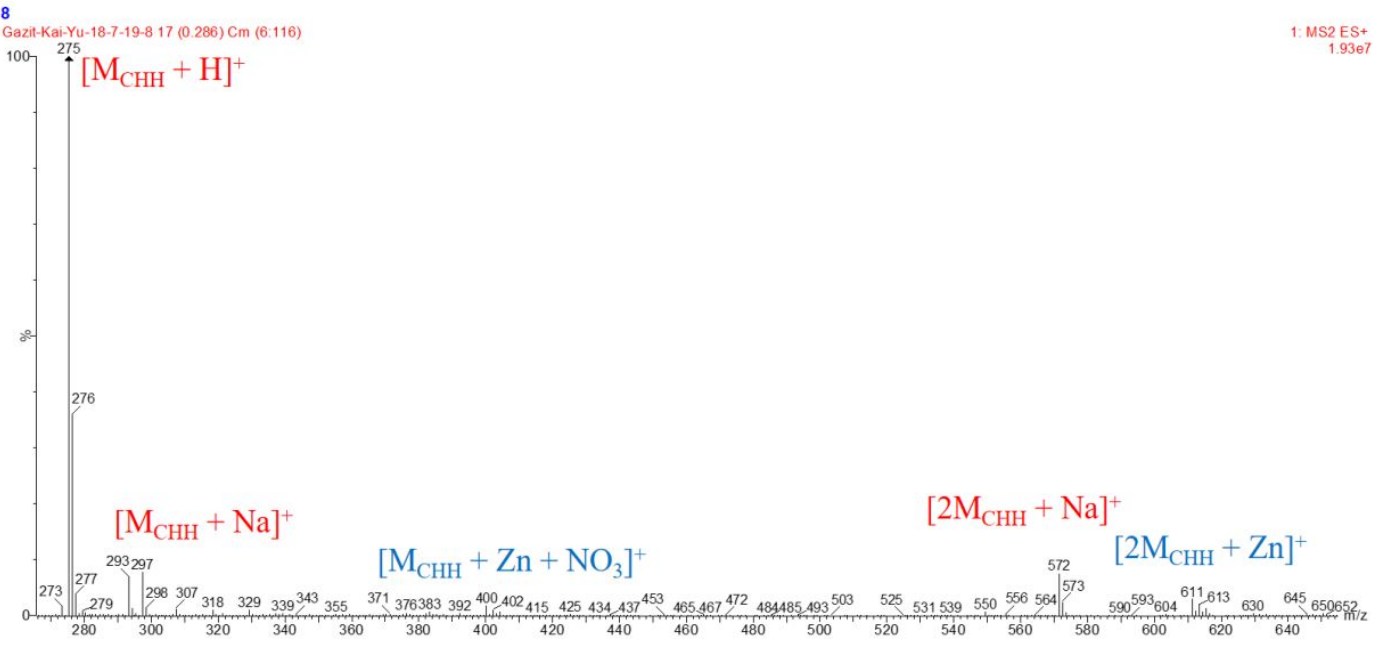

Supplementary Figure S10. Mass spectra of CHH and CHH-Zn, showing the m/z 400 and 611.2 corresponding to the oligomer of (a) $\left[\mathrm{M}_{\mathrm{CHH}}+\mathrm{Zn}+\mathrm{NO}_{3}\right]$ and (b) $\left[2 \mathrm{M}_{\mathrm{CHH}}+\mathrm{Zn}\right]$. 


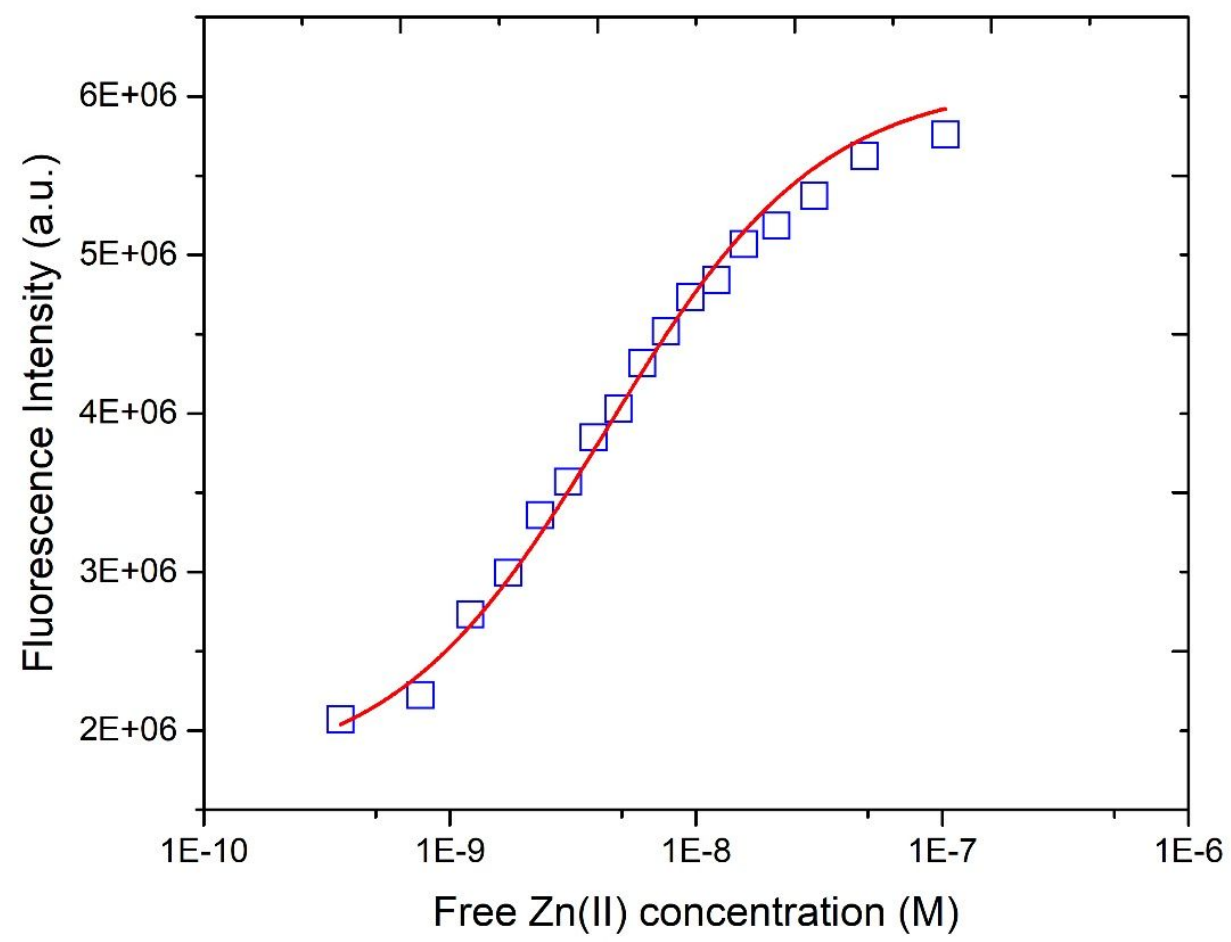

Supplementary Figure S11. Fluorescence intensity $\left(\lambda_{\mathrm{ex}}=380 \mathrm{~nm}, \lambda_{\mathrm{em}}=500 \mathrm{~nm}\right)$ of CHH as a function of free $\mathrm{Zn}$ (II) concentration. The fluorescence intensity data were fitted with 1:1 binding model, according the reported method ${ }^{25}$.

$$
F=\frac{\left[Z n^{2+}\right]_{\text {free }} F_{\text {max }}+K_{d} F_{\text {min }}}{K_{d}+\left[Z n^{2+}\right]_{\text {free }}}
$$

Where $F$ is fluorescence intensity, $K_{\mathrm{d}}$ is dissociation constant, $F_{\min }$ is fluorescence intensity of free ligand, $F_{\max }$ is fluorescence intensity of $\mathrm{Zn}(\mathrm{II})$ loaded and $\left[\mathrm{Zn}^{2+}\right]_{\text {free }}$ is the free $\mathrm{Zn}^{2+}$ concentration. The values of apparent dissociation constants of $\mathrm{CHH}$ with $\mathrm{Zn}$ (II) was $4.33 \pm 0.13 \mathrm{nM}$. 

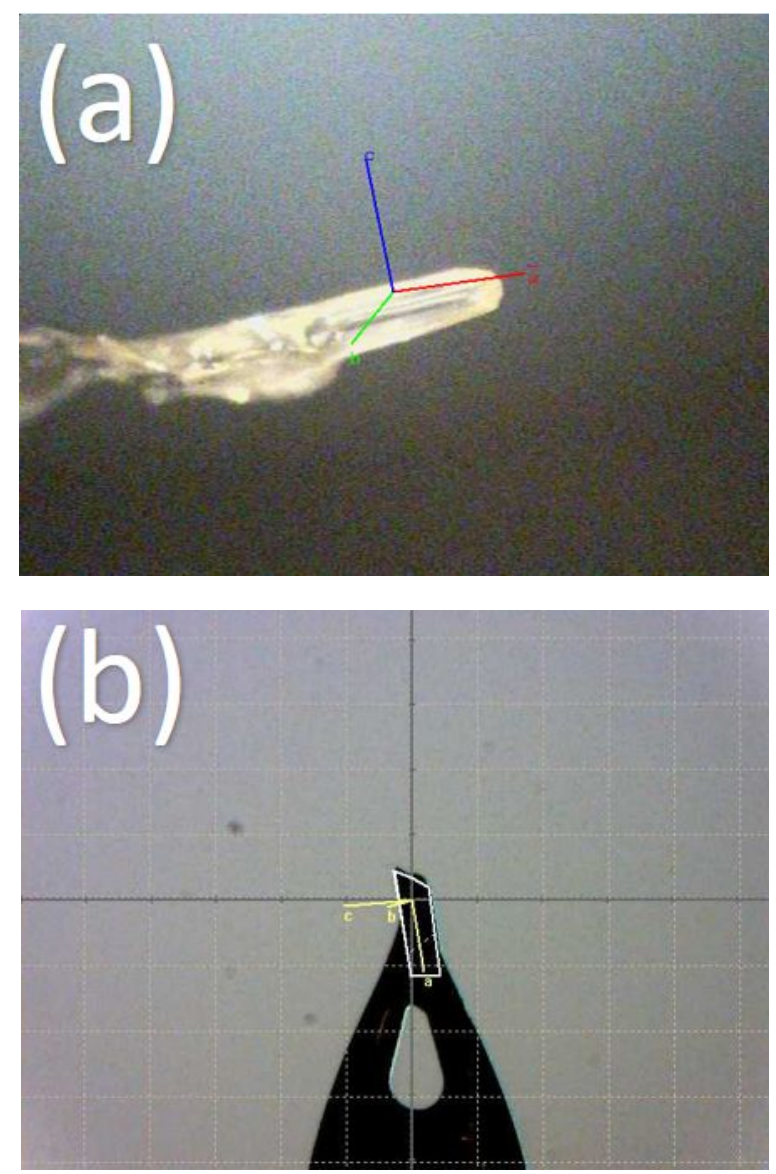

Supplementary Figure S12. Unit cell measurement of the single crystal of (a) $\mathrm{CHH}-\mathrm{Zn}\left(\mathrm{NO}_{3}\right)_{2}$ and (b) $\mathrm{CHH}-\mathrm{NaNO}_{3}$ with respect to the needle- and plaque-shaped crystal morphology, respectively. Single crystal is shown mounted on a MiTeGen loop. The crystal is highlighted in the center of the photo and the cell axes are shown. 


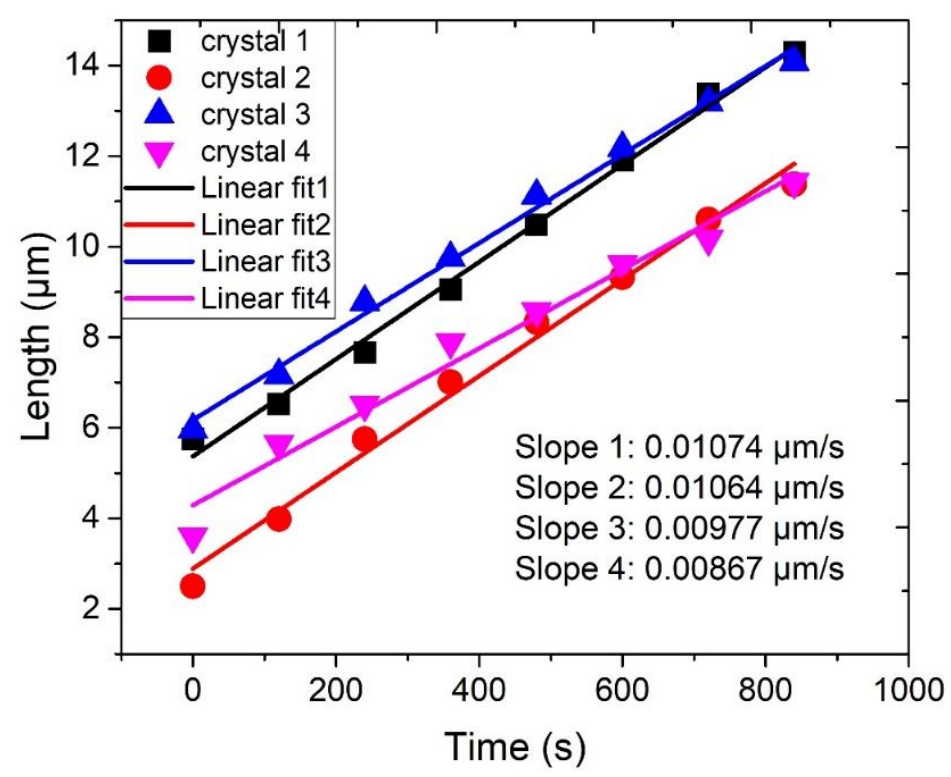

Supplementary Figure S13. Graphical representation of the elongation rate of 4 points taken from the growth of CHH-Zn( $\left(\mathrm{NO}_{3}\right)_{2}$ crystal in Supplementary Movie S1 and Figure 2c in the main text. The average measured elongation rate of $\mathrm{CHH}-\mathrm{Zn}\left(\mathrm{NO}_{3}\right)_{2}$ single crystal is $0.01 \mu \mathrm{m} \mathrm{s}^{-1}$. 


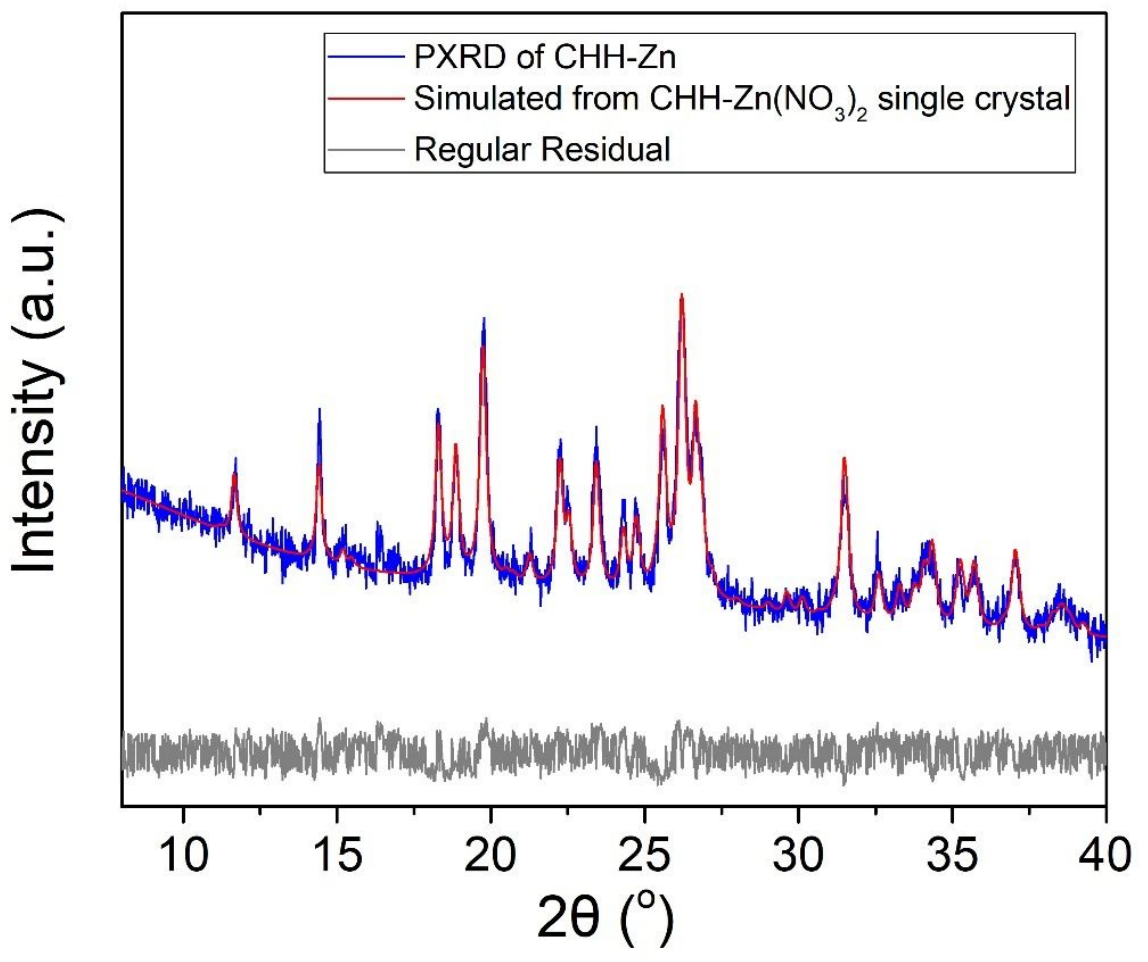

Supplementary Figure S14. Fitting of the observed PXRD of CHH-Zn (blue) and calculated diffractogram of $\mathrm{CHH}-\mathrm{Zn}\left(\mathrm{NO}_{3}\right)_{2}$ single crystal (red). The difference between the diffractograms is represented by a grey line. 


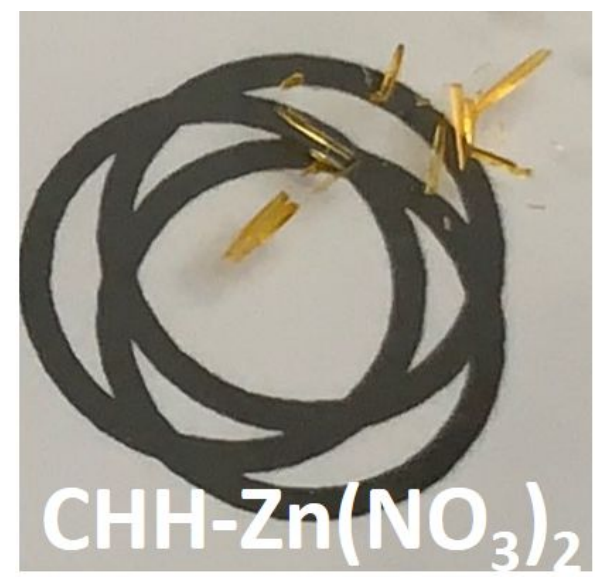

Supplementary Figure S15. Optical image of CHH- $\mathrm{Zn}\left(\mathrm{NO}_{3}\right)_{2}$ single crystals. 

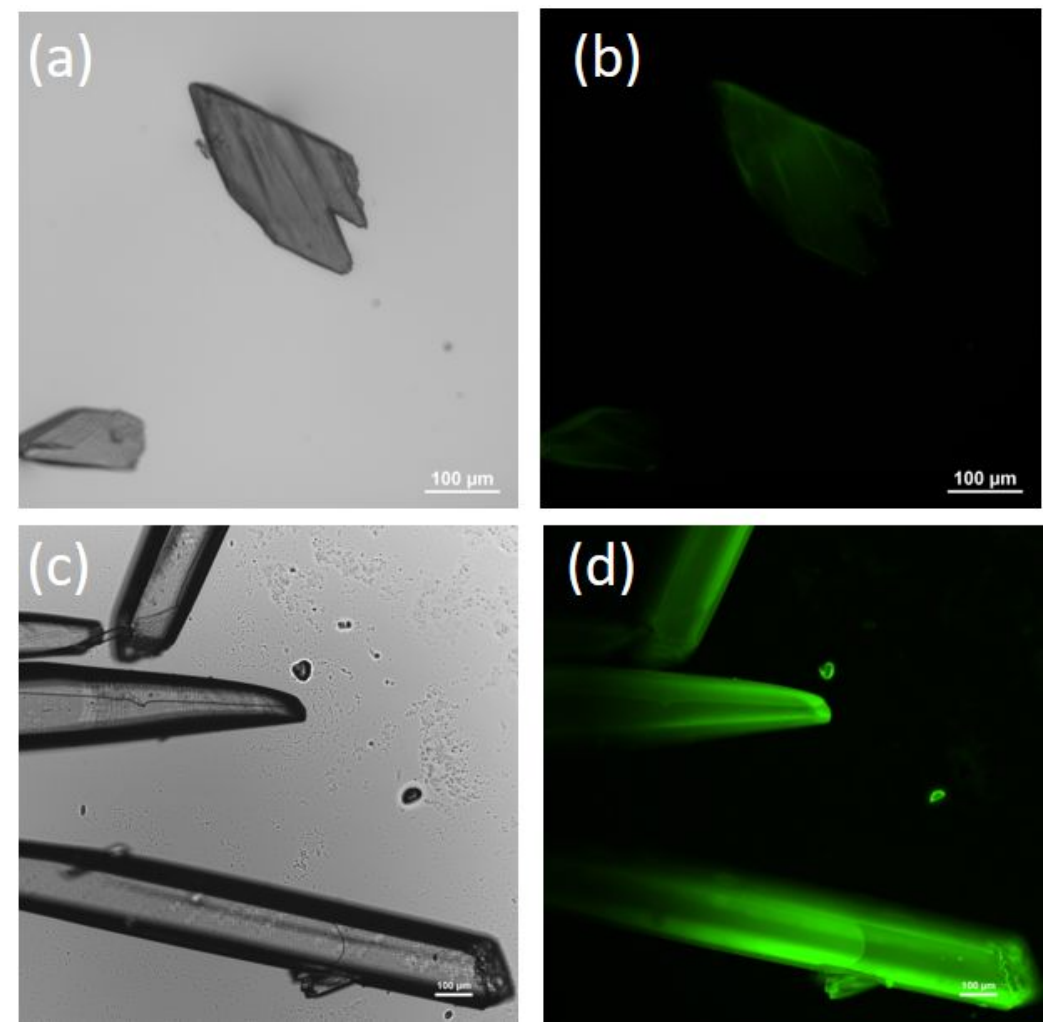

Supplementary Figure S16. Microscopy images of the (a, b) $\mathrm{CHH}_{-} \mathrm{NaNO}_{3}$ and (c, d) $\mathrm{CHH}-$ $\mathrm{Zn}\left(\mathrm{NO}_{3}\right)_{2}$ single crystals. (a, c) Bright field. (b, d) Fluorescence images acquired with excitation and emission filters of $405 \mathrm{~nm}$ and $525 \mathrm{~nm}$, respectively, and exposure time of $200 \mathrm{~ms}$. 


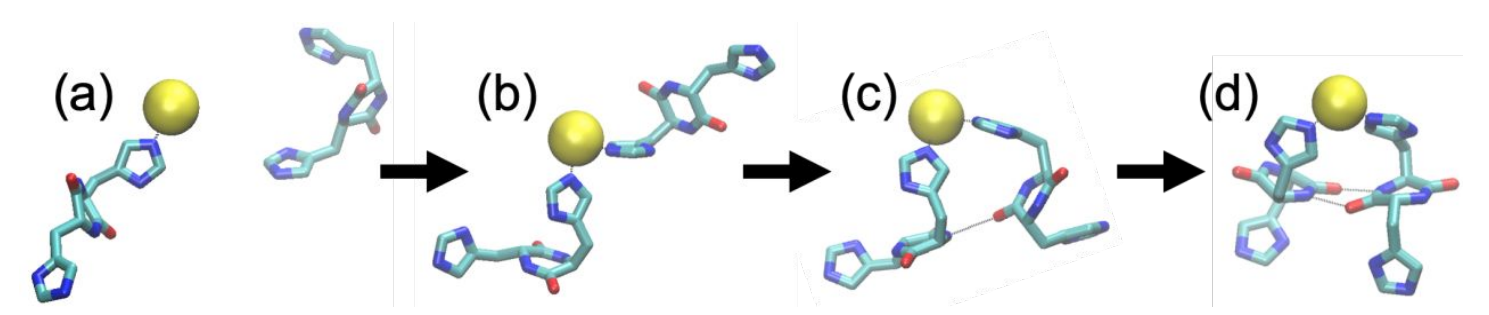

Supplementary Figure S17. Molecular graphics image of the coordination induced selfassembly of $\mathrm{CHH}$ with $\mathrm{Zn}(\mathrm{II})$. The $\mathrm{CHH}$ are shown in licorice representation. Zinc ions are shown in gray VDW representation. Hydrogen bonds and zinc coordination are indicated with black dotted lines. In this case, $\mathrm{Zn}(\mathrm{II})$ first coordinates with the imidazole ring of $\mathrm{CHH}$ (a). Then, another imidazole ring of second $\mathrm{CHH}$ coordinates with $\mathrm{Zn}(\mathrm{II})$ (b). With the $\mathrm{Zn}(\mathrm{II})$ coordinated with two histidine of opposing $\mathrm{CHH}$ simultaneously and thus stabilizing the two $\mathrm{CHH}$, the $\mathrm{CHH}$ begins forming hydrogen bond interactions with each other (c) to finally form $\beta$-bridge interactions (d). The presented structures were extracted from simulations of $\mathrm{CHH}$ and $\mathrm{Zn}\left(\mathrm{NO}_{3}\right)_{2}$, but are also observed within simulations of $\mathrm{CHH}$ and $\mathrm{ZnCl}_{2}$. 

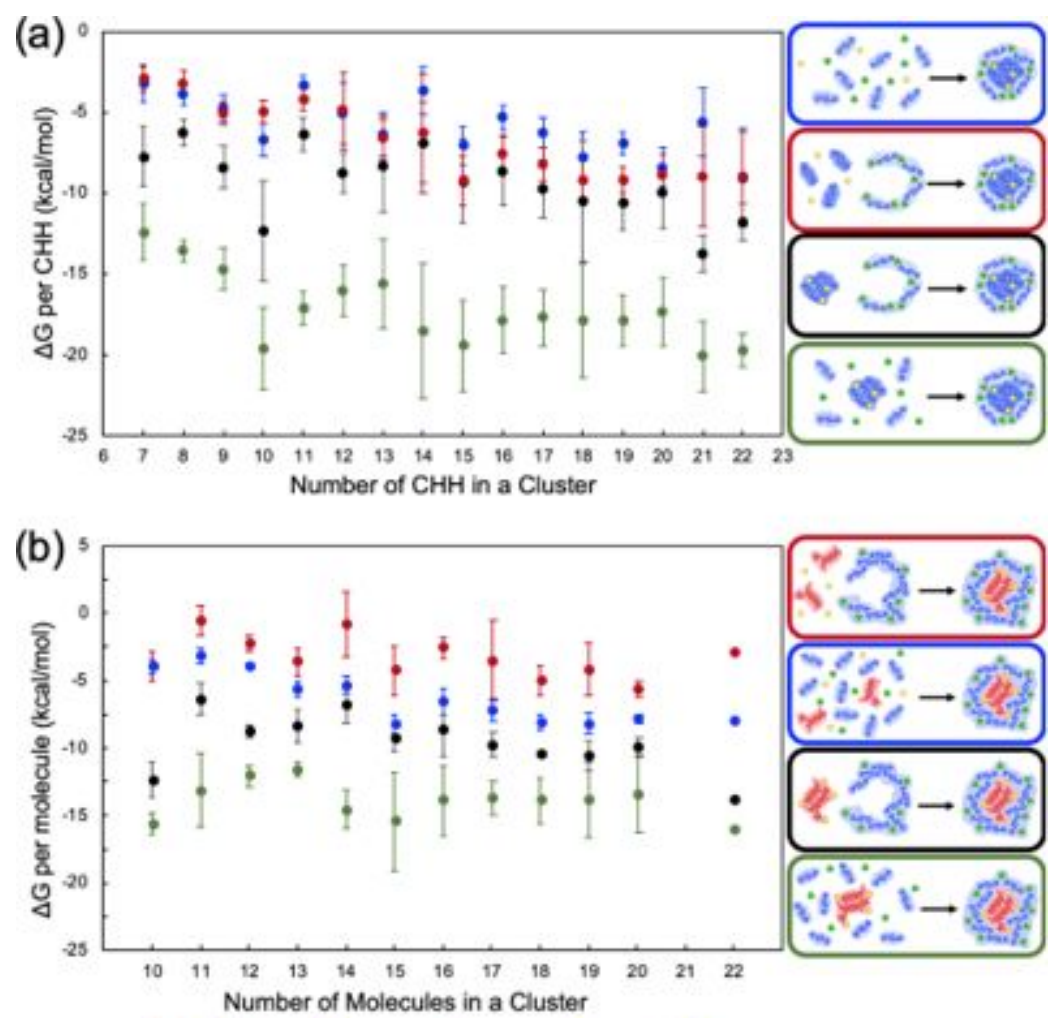

(c)
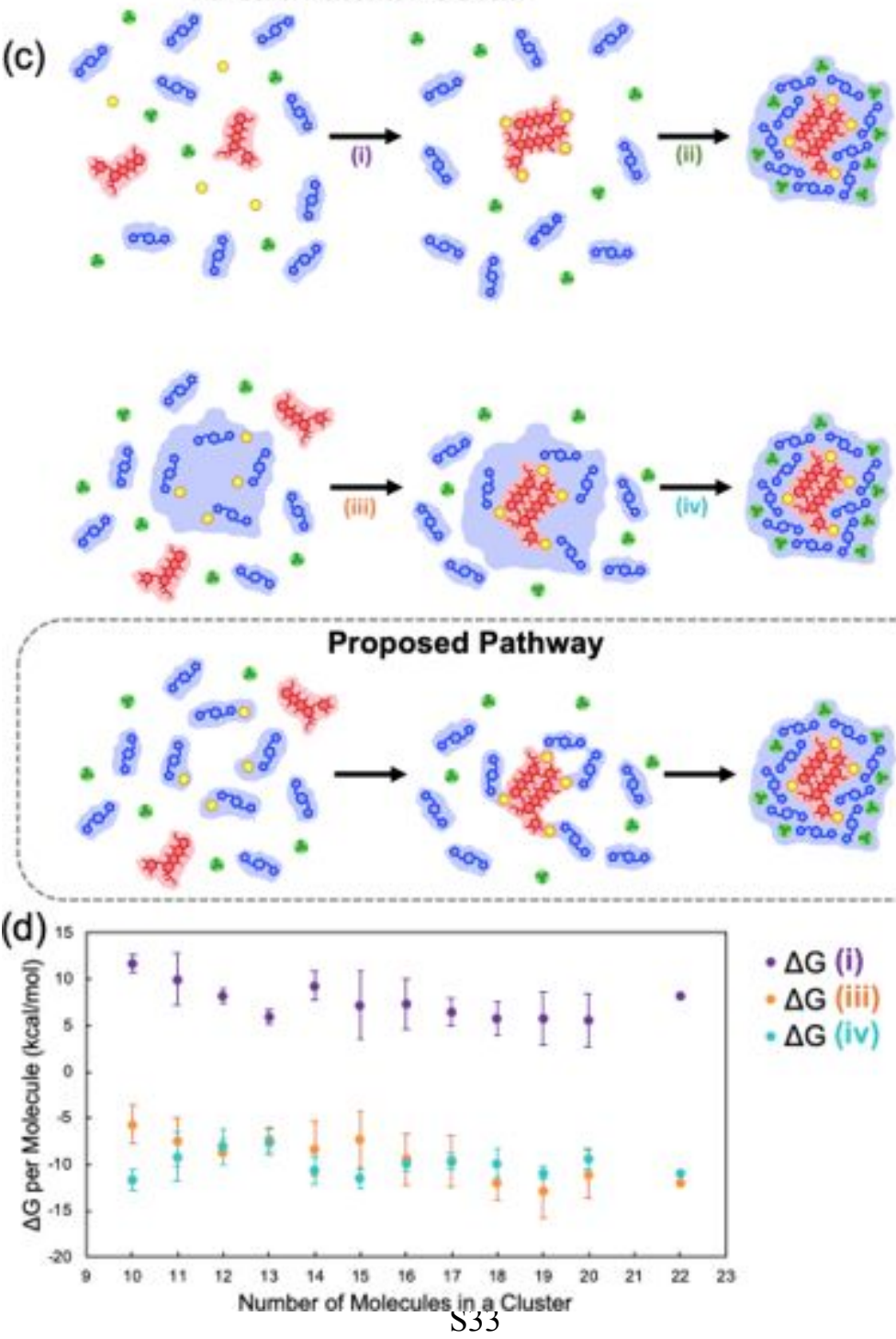
Supplementary Figure S18. Free energy to associate the clusters of (a) $\mathrm{CHH}-\mathrm{Zn}(\mathrm{II})-\mathrm{NO}_{3}{ }^{-}$or (b) $\mathrm{CHH}-\mathrm{Zn}(\mathrm{II})-\mathrm{NO}_{3}{ }^{-}+\mathrm{EPI}$ through different pathways, shown as schematic illustrations in the right panel. The free energy to associate the clusters was calculated, (blue) by assuming that the individual entities forming the cluster spontaneously self-assemble into a cluster, (black) by assuming that the interior nucleus and the exterior surface assemblies are preformed and subsequently aggregate to form a cluster, (red) by assuming that the exterior surface assembly is formed first and individual entities forming the interior nucleus aggregate on the preformed exterior surface to form a cluster, and (green) by assuming that the interior nucleus assembly is formed first and individual entities forming the exterior surface wrap around the preformed nucleus to form a cluster. (c) Schematic of the potential pathways related to the most energetically favorable pathway according to (b, ii). The free energy to (i) associate the EPI+Zn(II) interior cluster from the individual entities is unfavorable. However, the free energy to (iii) associate the $\mathrm{EPI}+\mathrm{Zn}$ (II) interior cluster is favorable if the $\mathrm{Zn}$ (II) ions are in a peptide-like environment prior to association, and the (iv) interior cluster is formed in a peptide-like environment of the cavity of the cluster prior to the formation of the cluster. We propose (encircled in grey dotted lines) that $\mathrm{Zn}$ (II) is pulled from the isopropanol environment into a more peptide-like environment by individual or pairs of $\mathrm{CHH}$, enabling the self-encapsulation of Epirubicin molecules first, forming the interior nucleus of the clusters, which further facilitates the assembly of individual pieces of $\mathrm{CHH}$ and $\mathrm{NO}_{3}{ }^{-}$exteriorly wrapping around the preformed Epirubicin interior. (d) Free energy to associate the intermediate clusters of $\mathrm{CHH}-\mathrm{Zn}(\mathrm{II})-\mathrm{NO}_{3}{ }^{-}+\mathrm{EPI}$ (purple, i) assuming that the individual entities forming the cluster are fully immersed in isopropanol alcohol prior to the formation of the interior cluster, (orange, iii) assuming that the $\mathrm{Zn}(\mathrm{II})$ are in the peptide-like environment of the cavity of the cluster prior to the formation of the interior cluster, and (cyan, iv) the free energy to associate the cluster of $\mathrm{CHH}-\mathrm{Zn}(\mathrm{II})-\mathrm{NO}_{3}{ }^{-}+\mathrm{EPI}$ assuming that the interior cluster is formed first in a peptide-like environment of the cavity of the cluster prior to the formation of the cluster. 

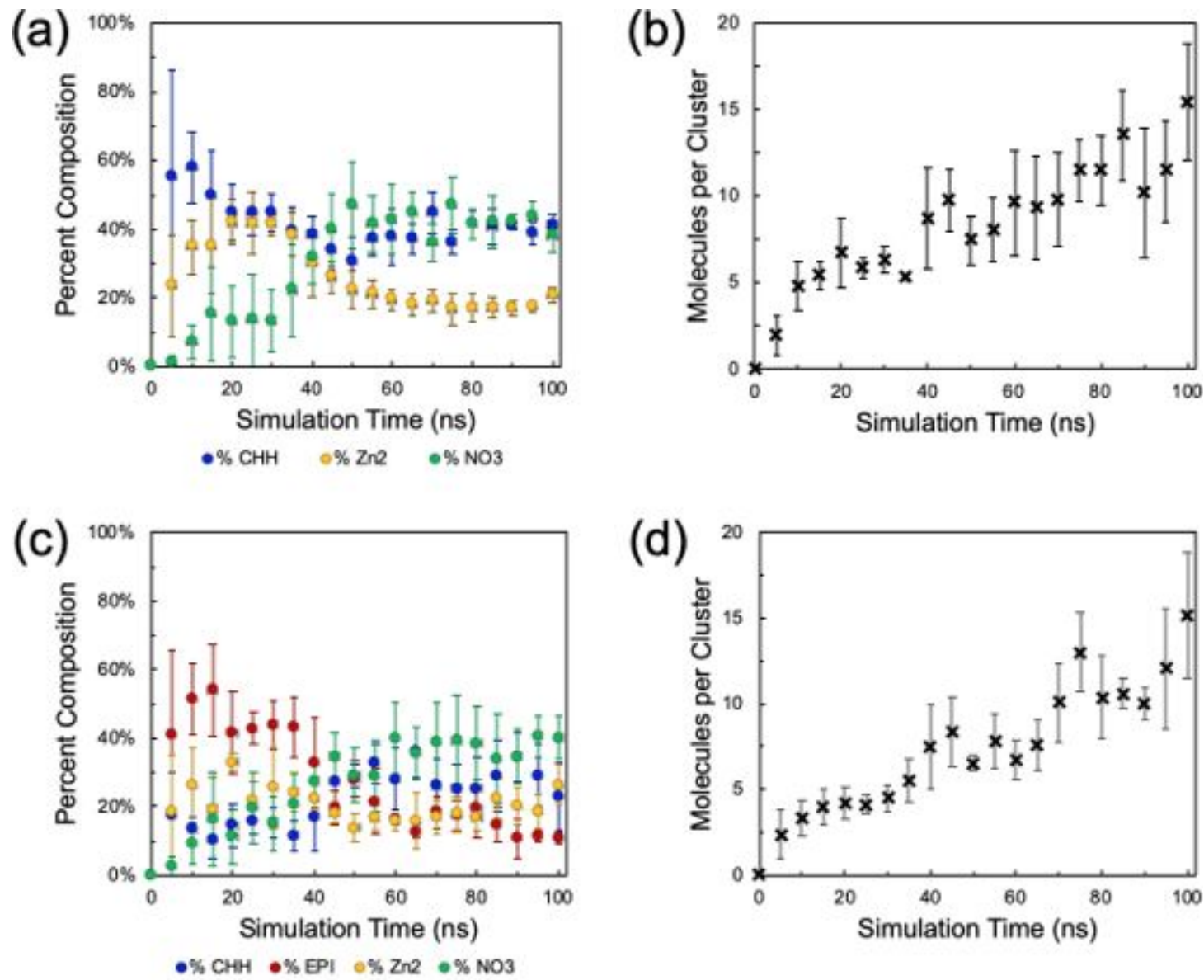

Supplementary Figure S19. Time evolution percent composition of clusters within (a-b) the simulation system of $\mathrm{CHH}-\mathrm{Zn}(\mathrm{II})-\mathrm{NO}_{3}{ }^{-}$, and (c-d) the simulations system of $\mathrm{CHH}-\mathrm{Zn}(\mathrm{II})-\mathrm{NO}_{3}{ }^{-}+\mathrm{EPI}$. (a) The time evolution percent composition was calculated for clusters composed of the entities $\left(\mathrm{CHH}, \mathrm{Zn}(\mathrm{II})\right.$, or $\mathrm{NO}_{3}^{-}$) that eventually form clusters containing at least $10 \mathrm{CHH}$ dipeptides. (b) molecules in the cluster as the function of simulation time in system of $\mathrm{CHH}-\mathrm{Zn}(\mathrm{II})-\mathrm{NO}_{3}^{-}$. (c) The time evolution percent composition was calculated for clusters composed of the entities $(\mathrm{CHH}$, $\mathrm{Zn}(\mathrm{II}), \mathrm{NO}_{3}{ }^{-}$or EPI) that eventually form clusters containing at least 10 molecules with a composition ranging from $53 \% \mathrm{CHH}$ and $83 \% \mathrm{EPI}$ to $47 \% \mathrm{CHH}$ and $17 \%$ EPI. (d) molecules in the cluster as the function of simulation time in system of $\mathrm{CHH}-\mathrm{Zn}(\mathrm{II})-\mathrm{NO}_{3}{ }^{-}+\mathrm{EPI}$. The plotted average and standard deviation percent composition and number of molecules per cluster correspond to the block averaged values in $5 \mathrm{~ns}$ increments. 

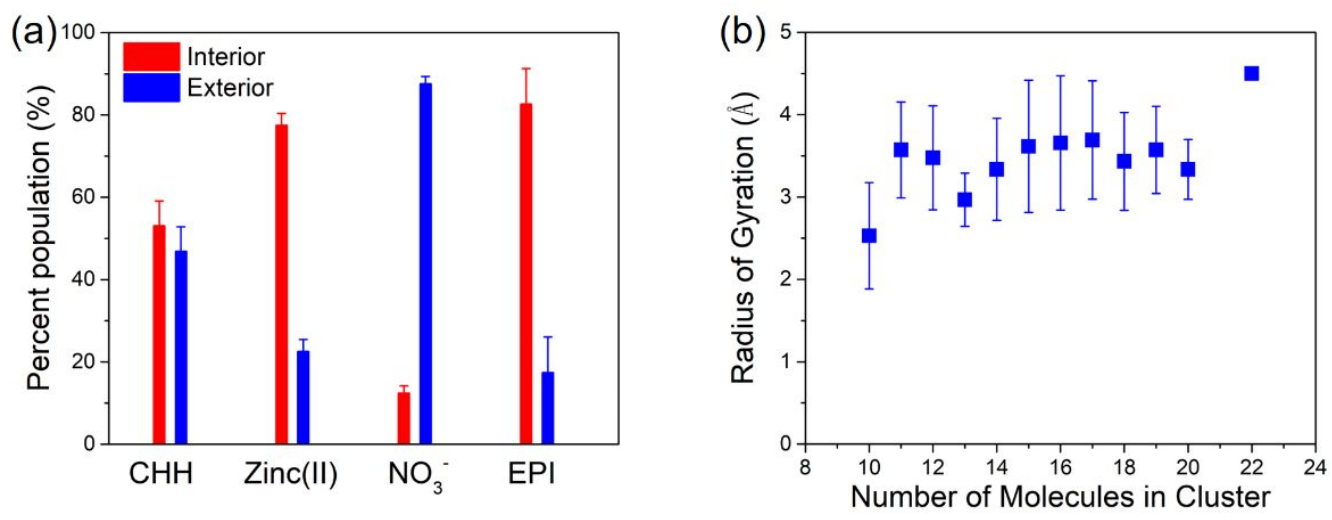

Supplementary Figure S20. Structural analysis of EPI encapsulation by $\mathrm{CHH}-\mathrm{Zn}(\mathrm{II})-\mathrm{NO}_{3}{ }^{-}$. (a) Percent population of $\mathrm{CHH}, \mathrm{EPI}, \mathrm{Zn}(\mathrm{II})$ and $\mathrm{NO}_{3}{ }^{-}$within the interior and exterior of the clusters containing at least 10 molecules with a composition ranging from $53 \% \mathrm{CHH}$ and $83 \%$ EPI to $47 \%$ $\mathrm{CHH}$ and $17 \%$ EPI. (b) Difference of gyration radius in $\AA$ between $\mathrm{CHH}$ and EPI within the clusters containing at least 10 molecules with a composition ranging from 53\% $\mathrm{CHH}$ and $83 \%$ EPI to $47 \%$ $\mathrm{CHH}$ and $17 \%$ EPI. A positive value indicates a larger radius of gyration in the $\mathrm{CHH}$ layer than in the EPI layer within a given cluster. 


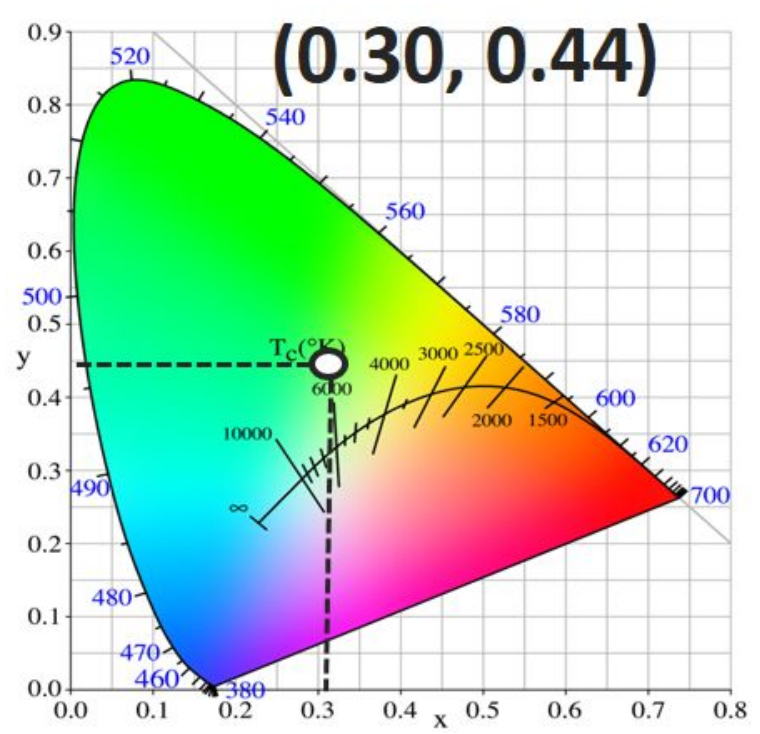

Supplementary Figure S21. CIE color coordinates of LED using CHH-Zn as a phosphor during operation 


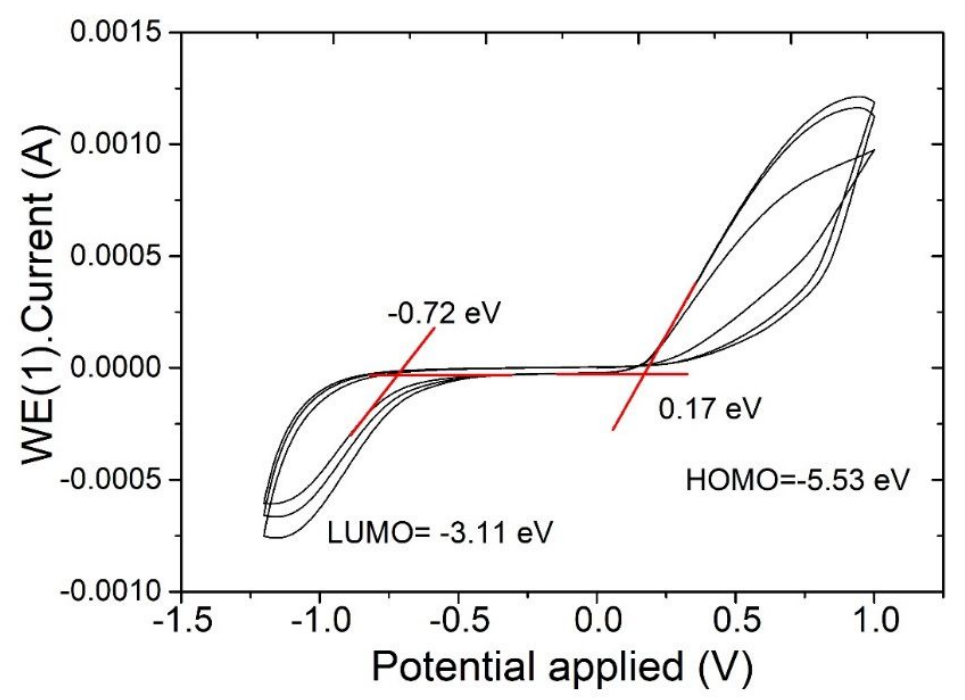

Supplementary Figure S22. Cyclic voltammetry traces for CHH-Zn. The calculated energy level of the highest occupied and lowest unoccupied molecular orbitals of CHH-Zn was -5.53 and -3.11 $\mathrm{eV}$, respectively. 

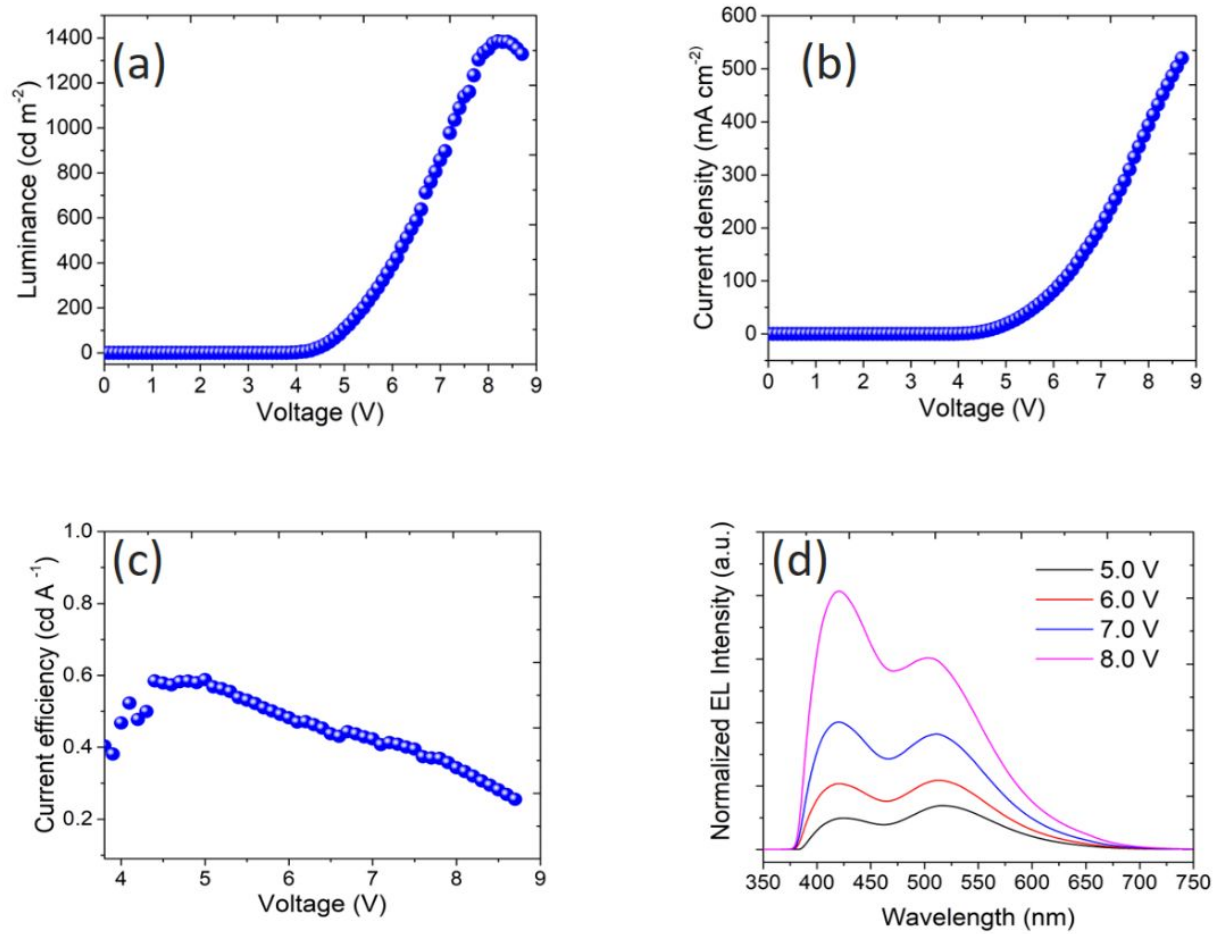

Supplementary Figure S23. (a) Luminance-current (L-V), (b) current-voltage (I-V), (c) current efficiency-voltage (CE-V), and (d) electroluminescence spectra at different bias voltage of CHHZn-based OLED. 


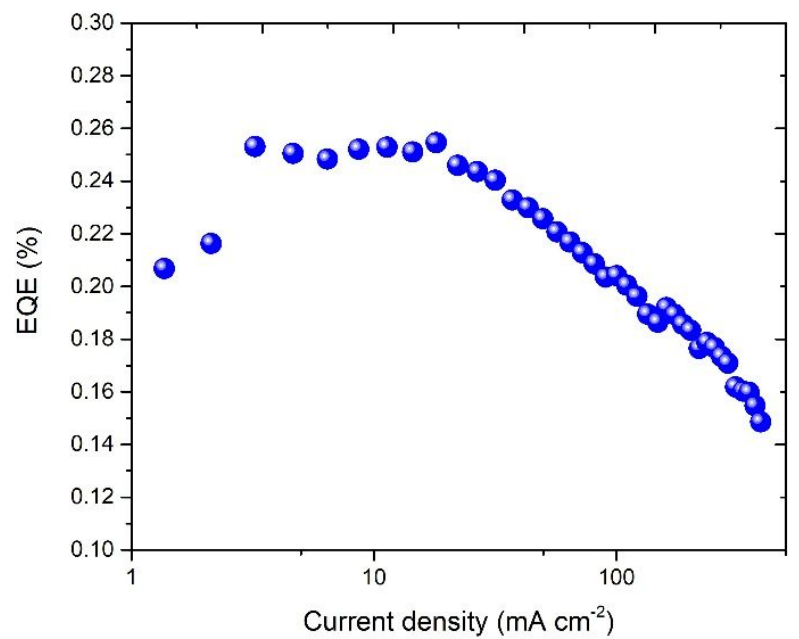

Supplementary Figure S24. EQE-current density of CHH-Zn-based OLED. 


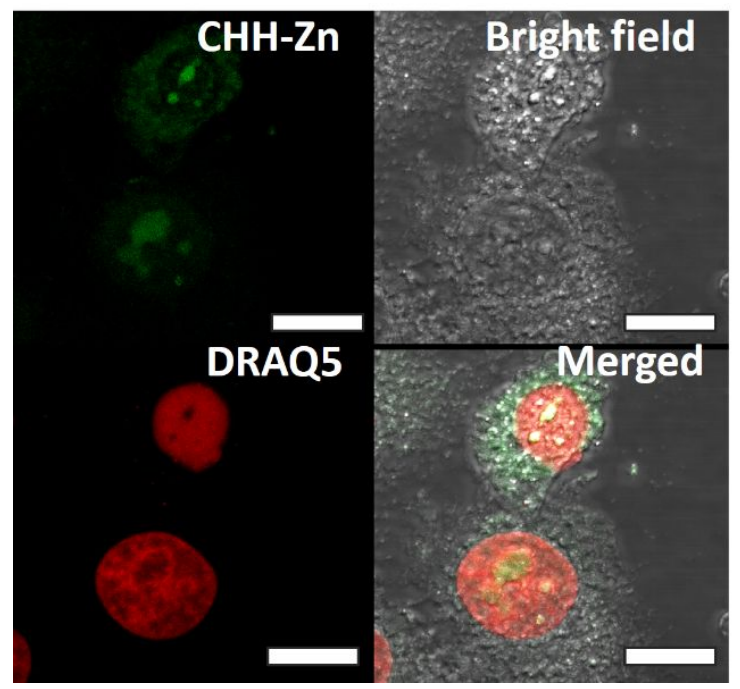

Supplementary Figure S25. Confocal microscopy imaging of live cells treated with CHH-Zn (3 $\mu \mathrm{g} / \mathrm{mL}$ ) and DRAQ5. Scale bar is $25 \mu \mathrm{m}$. 


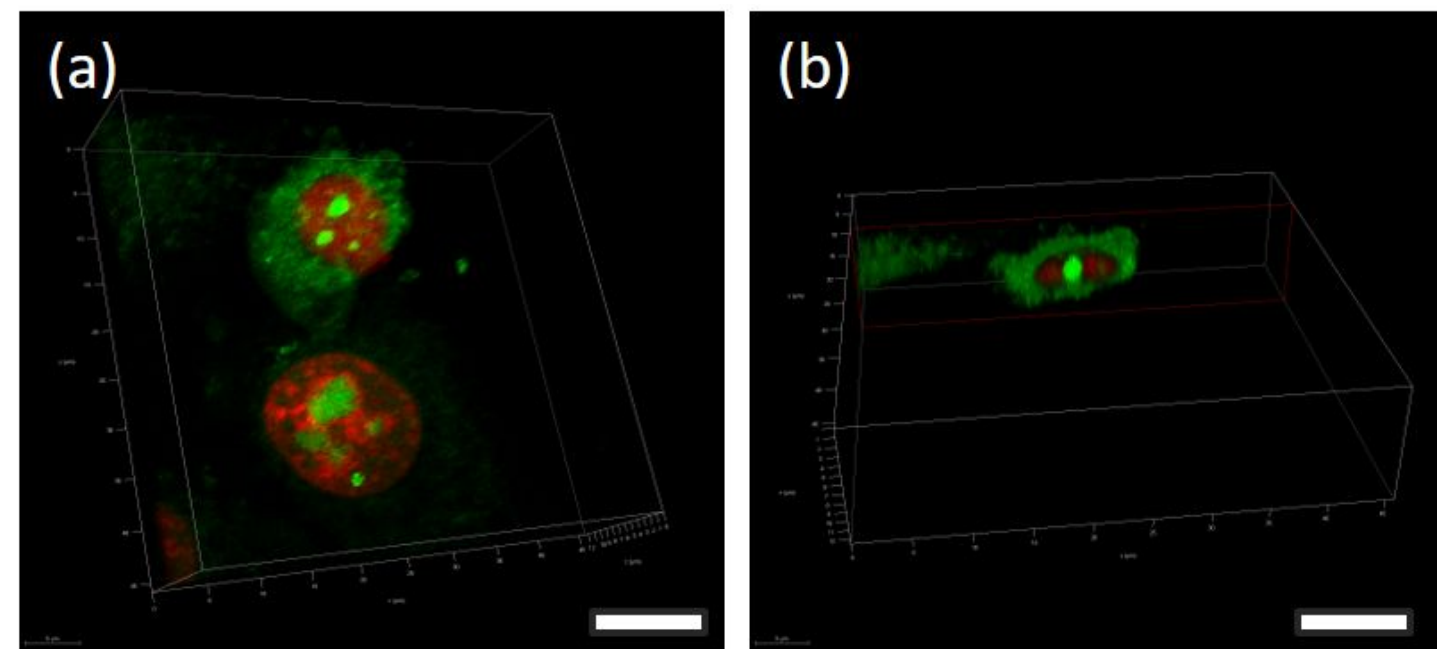

Supplementary Figure S26. Confocal microscopy imaging of live cells treated with CHH-Zn (3 $\mu \mathrm{g} / \mathrm{mL}$ ) and DRAQ5. (a) Z-Stack 3D image of HeLa cells and (b) the XY section image visualized through $3 \mathrm{D}$ reconstruction. 


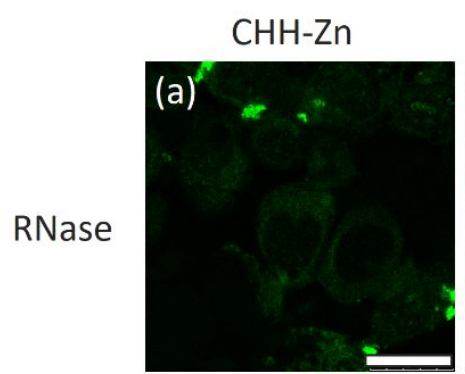

EPI
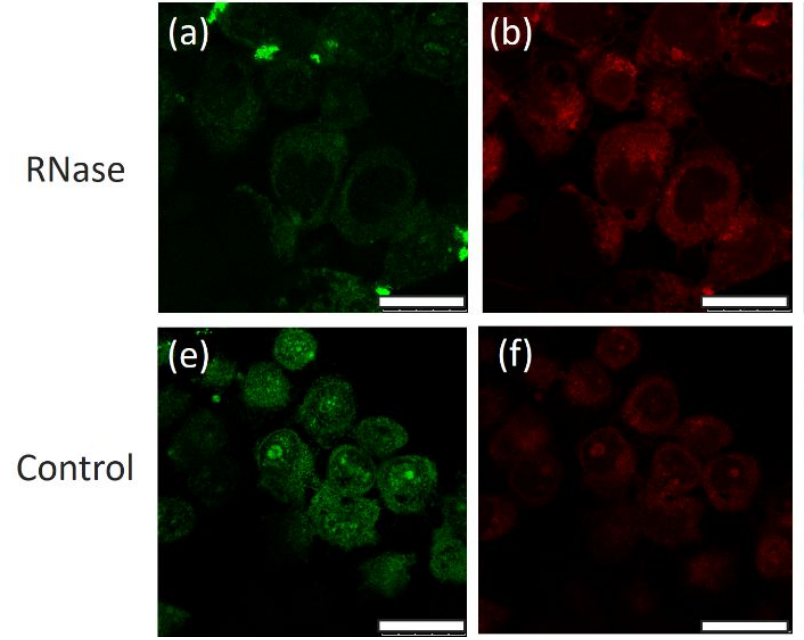

DRAQ5
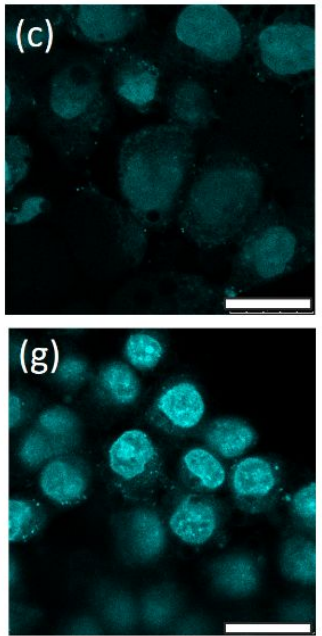

Merged
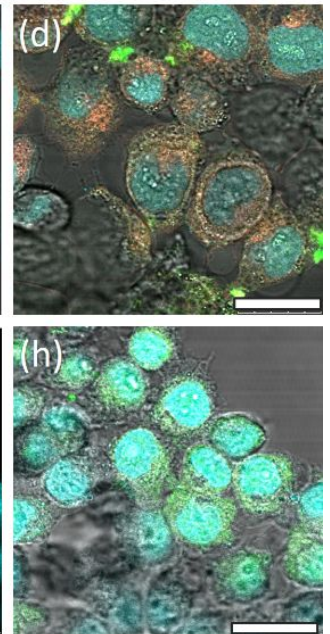

Supplementary Figure S27. (a-h) Confocal fluorescence images of (a, e) CHH-Zn, (b, f) EPI and (c, g) DRAQ5 with ("RNase") and without ("control") prior ribonuclease (RNase) treatment of HeLa cells $(\mathrm{CHH}-\mathrm{Zn}, \lambda \mathrm{ex}=405 \mathrm{~nm}, \lambda \mathrm{em}=420-500 \mathrm{~nm}$; EPI, $\lambda \mathrm{ex}=543 \mathrm{~nm}, \lambda \mathrm{em}=550-750 \mathrm{~nm}$; DRAQ5, $\lambda$ ex $=633 \mathrm{~nm}, \lambda \mathrm{em}=750-780 \mathrm{~nm}$; scale bar $=25 \mu \mathrm{m}$ ). The RNase treatment was performed to demonstrate the disappearance of the bright $\mathrm{CHH}-\mathrm{Zn}$ fluorescence spots upon RNA degradation. However, no obvious effect of DRAQ5 staining results was found after digestion with RNase, as DRAQ5 preferably stains DNA rather than RNA. These results confirm that the CHH$\mathrm{Zn}+\mathrm{EPI}$ has a high affinity to the RNA-rich nucleolus. 


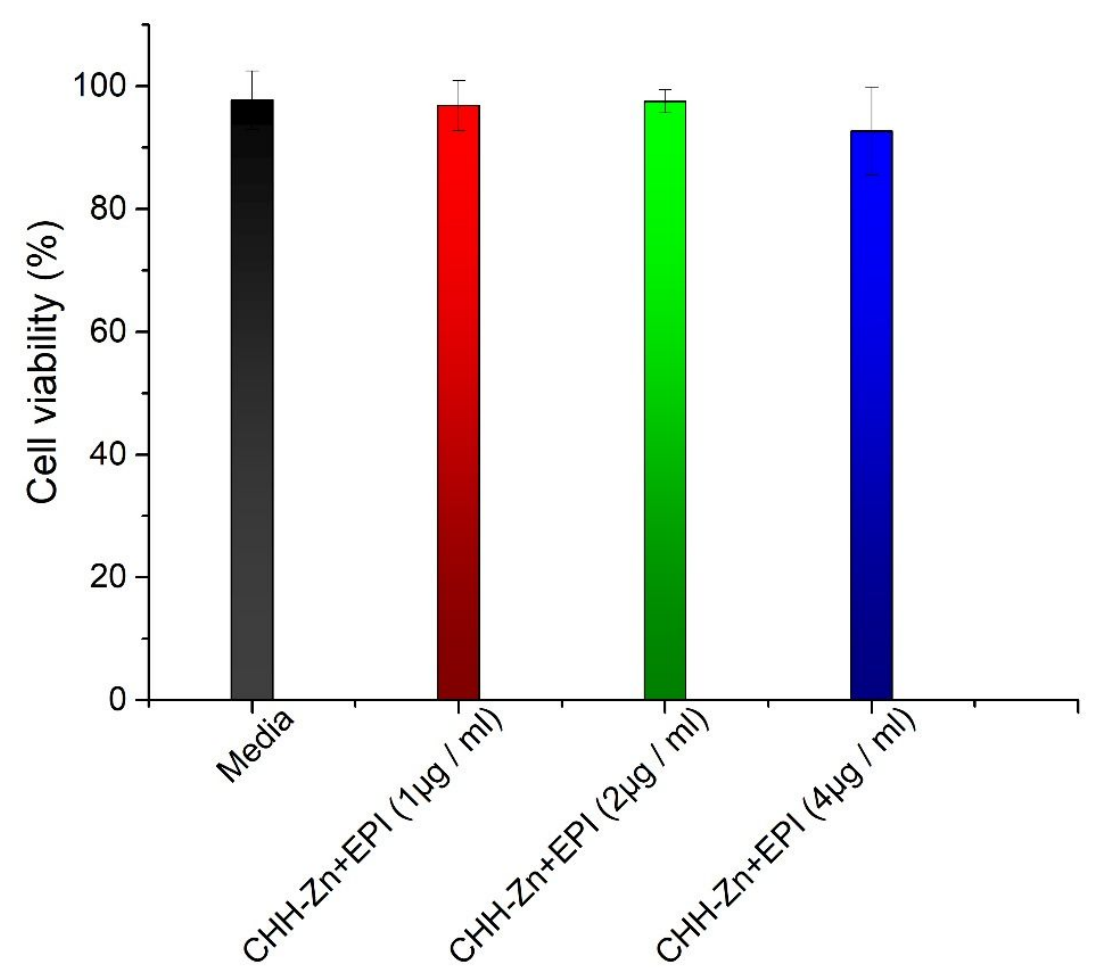

Supplementary Figure S28. HeLa cells viability following incubation with different concentrations of the CHH-Zn+EPI carrier $\left(0-4 \mu \mathrm{g} \mathrm{mL}^{-1}\right)$ for 12 hours. 


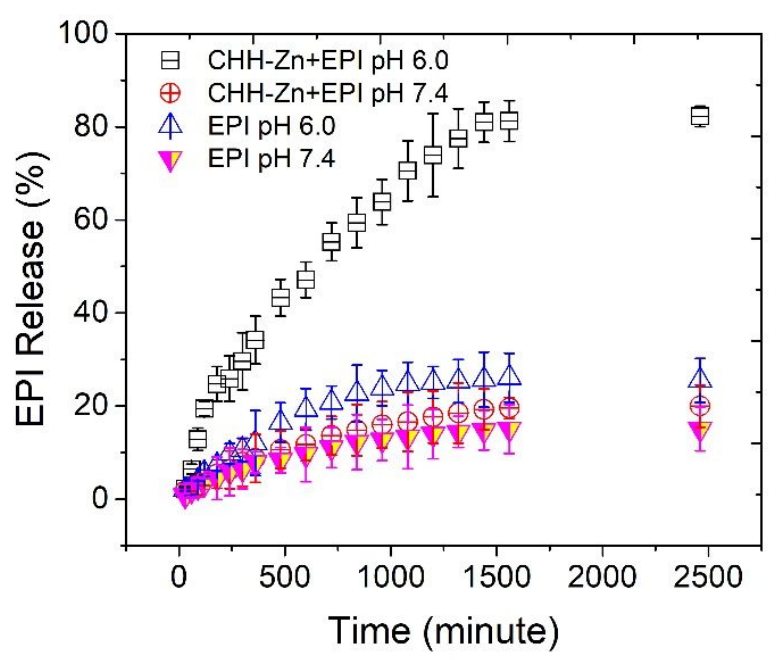

Supplementary Figure S29. Profiles of Epirubicin release from CHH-Zn+EPI and EPI alone in $3.5 \mathrm{kDa}$ dialysis chambers with different $\mathrm{pH}$ values ( $\mathrm{pH} 6.0$, or 7.4). 


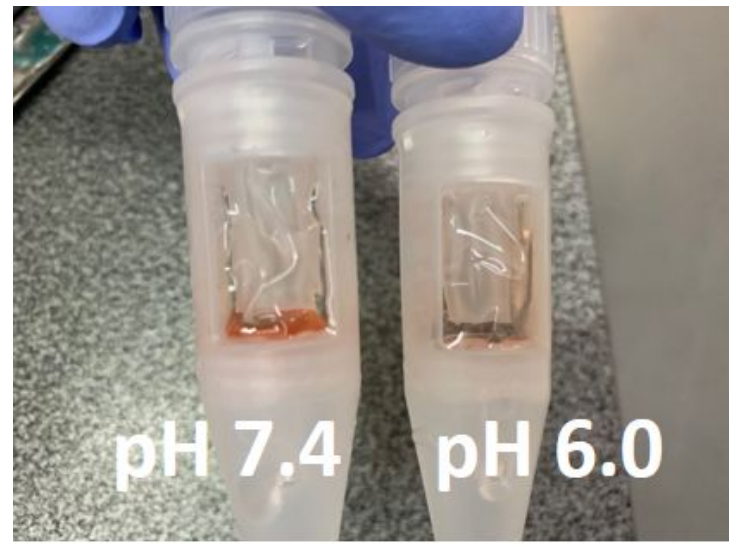

Supplementary Figure S30. The image of dialysis tube of CHH-Zn+EPI (left: at pH 7.4) and (right: at $\mathrm{pH} 6$ ) after 40 hours. 

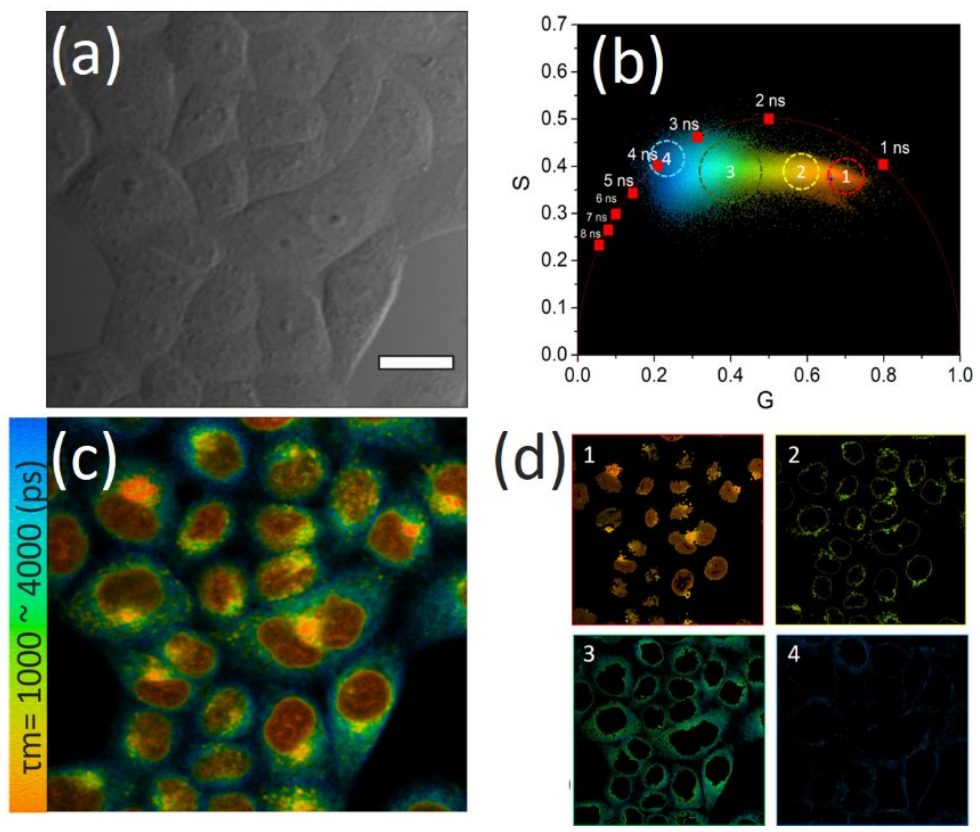

Supplementary Figure S31. Phasor-FLIM analysis of HeLa cells cultured with CHH-Zn+EPI at a concentration of $4 \mu \mathrm{g} / \mathrm{mL}$ for 4 hours. (a) Bright filed, (b) FLIM image, and (c) phasor-separated FLIM images of HeLa cells. (d) HeLa cells were divided into (1) nucleus, (2) nucleus membrane, (3) cytoplasm and (4) cell membrane according to the different fluorescence lifetimes of EPI in these four regions. 

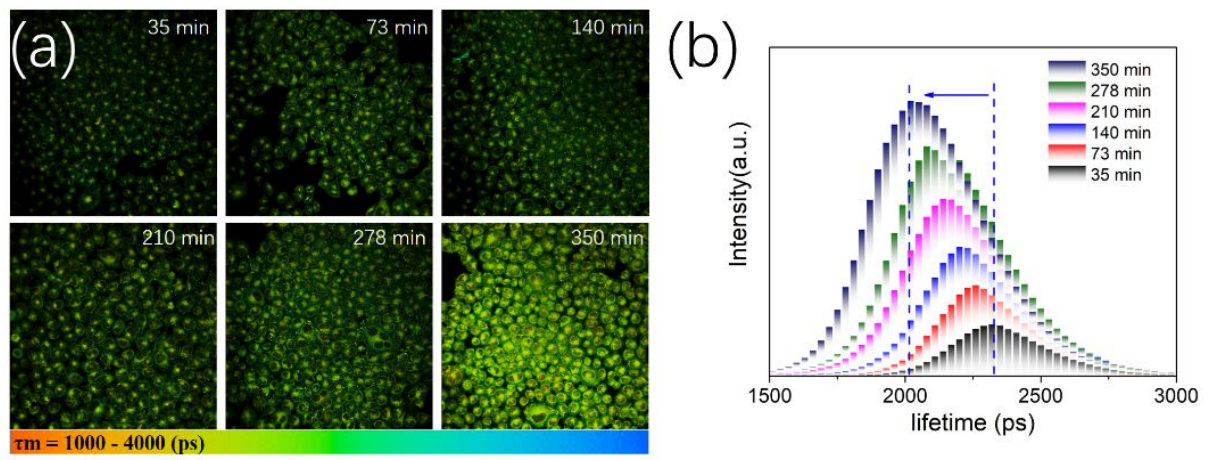

Supplementary Figure S32. (a) Two-photons FLIM analysis of CHH-Zn $(4 \mu \mathrm{g} / \mathrm{mL})$ fluorescent signal of HeLa cells after incubation with CHH-Zn+EPI for 35 min, 73 min, 140 min, 210min, 278 min, and $350 \mathrm{~min}$, as indicated, under excitation of $900 \mathrm{~nm}$. (b) Fluorescence lifetime histogram of CHH-Zn at different time points. 


\section{Supplementary Tables}

Table S1. Data collection and refinement statistics of CHH-ZnI $2, \mathrm{CHH}-\mathrm{NaNO}_{3}$ and $\mathrm{CHH}-\mathrm{Zn}\left(\mathrm{NO}_{3}\right)_{2}$ crystals.

\begin{tabular}{|c|c|c|c|}
\hline Crystal data & $\mathrm{CHH}-\mathrm{ZnI}_{2}$ & $\mathrm{CHH}-\mathrm{NaNO}_{3}$ & $\mathrm{CHH}-\mathrm{Zn}\left(\mathrm{NO}_{3}\right)_{2}$ \\
\hline Chemical formula & C15 H22 I2 N6 O3 Zn & C12 H16 N8 O8 & C12 H16 N8 O8 \\
\hline$M r$ & 653.58 & 400.33 & 400.33 \\
\hline Crystal system & Orthorhombic & Monoclinic & Monoclinic \\
\hline Space group & $P b c n$ & $P 2_{1} / \mathrm{c}$ & $P 2_{1} / \mathrm{c}$ \\
\hline$a(\AA)$ & $18.6751(8)$ & $6.15595(12)$ & $6.1690(13)$ \\
\hline$b(\AA)$ & $11.9733(5)$ & $15.6585(3)$ & $15.739(3)$ \\
\hline$c(\AA)$ & $21.1751(15)$ & $8.45332(12)$ & $8.4643(18)$ \\
\hline$\alpha\left(^{\circ}\right)$ & 90 & 90 & 90 \\
\hline$\beta\left(^{\circ}\right)$ & 90 & $93.2015(14)$ & $93.257(4)$ \\
\hline$\gamma\left({ }^{\circ}\right)$ & 90 & 90 & 90 \\
\hline$V\left(\AA_{3}\right)$ & 4734.8 & 813.57 & 820.5 \\
\hline$Z, Z$ & 8 & 2 & 2 \\
\hline$\mu\left(\mathrm{mm}^{-1}\right)$ & 3.670 & 1.206 & 0.138 \\
\hline Temperature (K) & 100 & 100 & 100 \\
\hline \multicolumn{4}{|l|}{ Data collection } \\
\hline Diffractometer & $\begin{array}{l}\text { Rigaku XtaLAB } \\
\text { Pro: Kappa dual } \\
\text { home/near }\end{array}$ & $\begin{array}{l}\text { Rigaku XtaLAB } \\
\text { Pro: Kappa dual } \\
\text { home/near }\end{array}$ & $\begin{array}{l}\text { Rigaku XtaLAB } \\
\text { Pro: Kappa dual } \\
\text { home/near }\end{array}$ \\
\hline Absorption correction & multi-scan & multi-scan & multi-scan \\
\hline$T \min , T \max$ & $0.561,0.693$ & $0.922,0.979$ & $0.977,0.989$ \\
\hline \multicolumn{4}{|l|}{ Refinement } \\
\hline$R[F 2>2 \sigma(F 2)]$ & 0.0404 & 0.0440 & 0.0474 \\
\hline$w R(F 2)$ & 0.1085 & 0.1171 & 0.1299 \\
\hline Goodness-of-fit & 1.034 & 0.928 & 1.048 \\
\hline No. of parameters & 266 & 127 & 127 \\
\hline $\mathrm{H}$-atom treatment & $\begin{array}{l}\text { H-atom parameters } \\
\text { constrained }\end{array}$ & $\begin{array}{l}\mathrm{H} \text {-atom parameters } \\
\text { constrained }\end{array}$ & $\begin{array}{l}\text { H-atom parameters } \\
\text { constrained }\end{array}$ \\
\hline
\end{tabular}


Table S2. Average $\mathrm{CHH}$ and $\mathrm{Zn}$ (II) ratio of $\mathrm{MD}$ simulation clusters comprising $\mathrm{CHH}-\mathrm{Zn}$ (II) (second column) and $\mathrm{CHH}-\mathrm{Zn}(\mathrm{II})-\mathrm{NO}_{3}{ }^{-}$(third column) per a cluster containing $n \mathrm{CHH}$ molecules (first column). The numbers of observed instances of a cluster containing $n \mathrm{CHH}$ molecules are presented in parenthesis.

\begin{tabular}{|l|l|l|}
\hline Number of $\mathrm{CHH}$ in a cluster & \multicolumn{1}{|c|}{$\mathrm{CHH}: \mathrm{Zn}(\mathrm{II})$ ratio (instances observed) } \\
\hline $\mathbf{7}$ & Presence of $\mathrm{NO}_{3}^{-}$ & Absence of $\mathrm{NO}_{3}^{-}$ \\
\hline $\mathbf{8}$ & $0.57 \pm 0.09(229)$ & $0.85 \pm 0.10(261)$ \\
\hline $\mathbf{9}$ & $0.52 \pm 0.10(251)$ & $0.75 \pm 0.21(245)$ \\
\hline $\mathbf{1 0}$ & $0.30 \pm 0.08(190)$ & $0.96 \pm 0.20(167)$ \\
\hline $\mathbf{1 1}$ & $0.41 \pm 0.07(108)$ & $0.82 \pm 0.16(98)$ \\
\hline $\mathbf{1 2}$ & $0.57 \pm 0.09(67)$ & $0.97 \pm 0.09(59)$ \\
\hline $\mathbf{1 3}$ & $0.40 \pm 0.13(61)$ & $0.89 \pm 0.11(63)$ \\
\hline $\mathbf{1 4}$ & $0.46 \pm 0.11(52)$ & $0.85 \pm 0.08(55)$ \\
\hline $\mathbf{1 5}$ & $0.29 \pm 0.26(59)$ & $0.82 \pm 0.09(40)$ \\
\hline $\mathbf{1 6}$ & $0.28 \pm 0.32(55)$ & $0.75 \pm 0.18(27)$ \\
\hline $\mathbf{1 7}$ & $0.50 \pm 0.09(42)$ & $0.79 \pm 0.25(13)$ \\
\hline $\mathbf{1 8}$ & $0.52 \pm 0.09(38)$ & $0.72 \pm 0.20(4)$ \\
\hline $\mathbf{1 9}$ & $0.57 \pm 0.21(25)$ & $0.89 \pm 0.00(1)$ \\
\hline $\mathbf{2 0}$ & $0.46 \pm 0.11(24)$ & N/A $(0)$ \\
\hline $\mathbf{2 1}$ & $0.50 \pm 0.10(13)$ & $0.79 \pm 0.10(3)$ \\
\hline $\mathbf{2 2}$ & $0.45 \pm 0.03(9)$ & N/A $(0)$ \\
\hline $\mathbf{2 3}$ & $0.48 \pm 0.10(2)$ & N/A (0) \\
\hline $\mathbf{2 4}$ & N/A $(0)$ & N/A (0) \\
\hline $\mathbf{2 5}$ & $0.29 \pm 0.00(1)$ & N/A (0) \\
\hline $\mathbf{2 6}$ & $0.44 \pm 0.00(1)$ & N/A (0) \\
\hline $\mathbf{2 7}$ & $0.55 \pm 0.02(3)$ & N/A (0) \\
\hline & $0.37 \pm 0.00(1)$ & N/A (0) \\
\hline
\end{tabular}


Table S3. Instances of antiparallel $\beta$-bridge conformations observed within clusters containing a given number of $\mathrm{CHH}$ molecules in the simulated $\mathrm{CHH}-\mathrm{Zn}(\mathrm{II})-\mathrm{NO}_{3}{ }^{-}$and $\mathrm{CHH}-\mathrm{Zn}$ (II) systems.

\begin{tabular}{|c|c|c|c|c|}
\hline \multirow{3}{*}{$\begin{array}{l}\text { Number of } \mathrm{CHH} \text { in a } \\
\text { cluster }\end{array}$} & \multicolumn{4}{|c|}{ Instances of antiparallel $\beta$-bridge conformations observed } \\
\hline & \multicolumn{2}{|c|}{ Presence of $\mathrm{NO}_{3}^{-}$} & \multicolumn{2}{|c|}{ Absence of $\mathrm{NO}_{3}^{-}$} \\
\hline & $\begin{array}{c}\text { 2-adjacent } \\
\mathrm{CHH}\end{array}$ & $\begin{array}{c}\text { 3-adjacent } \\
\mathrm{CHH}\end{array}$ & $\begin{array}{c}\text { 2-adjacent } \\
\mathrm{CHH}\end{array}$ & $\begin{array}{c}\text { 3-adjacent } \\
\mathrm{CHH}\end{array}$ \\
\hline 7 & 14 & 6 & 19 & 5 \\
\hline 8 & 16 & 3 & 12 & 11 \\
\hline 9 & 5 & 1 & 9 & 4 \\
\hline 10 & 8 & 4 & 7 & 0 \\
\hline 11 & 3 & 1 & 5 & 2 \\
\hline 12 & 3 & 0 & 8 & 0 \\
\hline 13 & 1 & 2 & 3 & 3 \\
\hline 14 & 3 & 1 & 1 & 1 \\
\hline 15 & 1 & 0 & 1 & 3 \\
\hline 16 & 0 & 0 & 2 & 0 \\
\hline 17 & 0 & 1 & 0 & 0 \\
\hline 18 & 1 & 0 & 0 & 1 \\
\hline 19 & 2 & 1 & N/A & N/A \\
\hline 20 & 0 & 0 & 0 & 1 \\
\hline 21 & 0 & 0 & N/A & N/A \\
\hline 22 & 0 & 0 & N/A & N/A \\
\hline 23 & N/A & N/A & N/A & N/A \\
\hline 24 & 0 & 0 & N/A & N/A \\
\hline 25 & 0 & 0 & N/A & N/A \\
\hline 26 & 0 & 1 & N/A & N/A \\
\hline 27 & 1 & 0 & N/A & N/A \\
\hline
\end{tabular}

Note: The first column corresponds to the number of $\mathrm{CHH}$ molecules a given cluster contains. The second and third columns correspond to the observed instance of antiparallel $\beta$-bridge conformations comprising 2-, or 3- adjacent peptides, respectively, observed within each type of cluster specified in column 1 in simulations performed in the presence of $\mathrm{NO}_{3}{ }^{-}$. The fourth and fifth columns correspond to the instances of antiparallel $\beta$-bridge conformations comprising 2- and 3adjacent peptides, respectively, observed within each type of cluster specified in column 1 in the simulations performed in the absence of $\mathrm{NO}_{3}{ }^{-}$. The sum of instances of antiparallel $\beta$-bridge conformations does not necessarily equal the total number of instances of a given cluster, as each cluster may comprise multiple instances of a $\beta$-bridge conformations (e.g., a cluster of $9 \mathrm{CHH}$ molecules may contain more than one pair of 2 adjacent $\mathrm{CHH}$ in antiparallel $\beta$-bridge conformation). 


\section{REFERENCES}

1. Arnon, Z. A.; Vitalis, A.; Levin, A.; Michaels, T. C. T.; Caflisch, A.; Knowles, T. P. J.; AdlerAbramovich, L.; Gazit, E. Dynamic Microfluidic Control of Supramolecular Peptide Self-Assembly. Nat. Commun. 2016, 7, 13190.

2. Tamamis, P.; Adler-Abramovich, L.; Reches, M.; Marshall, K.; Sikorski, P.; Serpell, L.; Gazit, E.; Archontis, G. Self-Assembly of Phenylalanine Oligopeptides: Insights from Experiments and Simulations. Biophys. J. 2009, 96, 5020-5029.

3. Wang, Y.; Latshaw, D. C.; Hall, C. K. Aggregation of A $\beta(17-36)$ in the Presence of Naturally Occurring Phenolic Inhibitors Using Coarse-Grained Simulations. J. Mol. Biol. 2017, 429, 38933908.

4. Jo, S.; Kim, T.; Iyer, V. G.; Im, W. CHARMM-GUI: A Web-Based Graphical User Interface for CHARMM. J. Comput. Chem. 2008, 29, 1859-1865.

5. Huang, J.; MacKerell, A. D. CHARMM36 All-Atom Additive Protein Force Field: Validation Based on Comparison to NMR Data. J. Comput. Chem. 2013, 34, 2135-2145.

6. Brooks, B. R.; Brooks, C. L., 3rd; Mackerell, A. D., Jr.; Nilsson, L.; Petrella, R. J.; Roux, B.; Won, Y.; Archontis, G.; Bartels, C.; Boresch, S.; Caflisch, A.; Caves, L.; Cui, Q.; Dinner, A. R.; Feig, M.; Fischer, S.; Gao, J.; Hodoscek, M.; Im, W.; Kuczera, K.; et al. CHARMM: The Biomolecular Simulation Program. J. Comput. Chem. 2009, 30, 1545-614.

7. Hoover, W. G. Canonical Dynamics: Equilibrium Phase-Space Distributions. Phys. Rev. A 1985, 31, 1695-1697.

8. Martyna, G. J.; Tobias, D. J.; Klein, M. L. Constant Pressure Molecular Dynamics Algorithms. J. Chem. Phys. 1994, 101, 4177-4189.

9. Ryckaert, J.-P.; Ciccotti, G.; Berendsen, H. J. C. Numerical Integration of the Cartesian Equations of Motion of a System with Constraints: Molecular Dynamics of $n$-Alkanes. J. Comput. Phys. 1977, 23, 327-341.

10. Seeber, M.; Cecchini, M.; Rao, F.; Settanni, G.; Caflisch, A. Wordom: A Program for Efficient Analysis of Mmolecular Dynamics Simulations. Bioinformatics 2007, 23, 2625-2627.

11. Seeber, M.; Felline, A.; Raimondi, F.; Muff, S.; Friedman, R.; Rao, F.; Caflisch, A.; Fanelli, F. Wordom: A User-Friendly Program for the Analysis of Molecular Structures, Trajectories, and Free Energy Surfaces. J. Comput. Chem. 2011, 32, 1183-1194.

12. Mayer, S. W. A Molecular Parameter Relationship between Surface Tension and Liquid Compressibility. J. Chem. Phys. 1963, 67, 2160-2164.

13. Tang, K. E. S.; Bloomfield, V. A. Excluded Volume in Solvation: Sensitivity of Scaled-Particle Theory to Solvent Size and Density. Biophys. J. 2000, 79, 2222-2234.

14. Hayes, J. M.; Archontis, G. MM-GB(PB)SA Calculations of Protein-Ligand Binding Free Energies. In Molecular Dynamics - Studies of Synthetic and Biological Macromolecules; Wang, L., Ed.; InTech: Rijeka, Croatia, 2012.

15. Gohlke, H.; Case, D. A. Converging Free Energy Estimates: MM-PB(GB)SA Studies on the Protein-Protein Complex Ras-Raf. J. Comput. Chem. 2004, 25, 238-250.

16. Im, W.; Lee, M. S.; Brooks Iii, C. L. Generalized Born Model with a Simple Smoothing Function. J. Comput. Chem. 2003, 24, 1691-1702.

17. M.T., K.; V.V., L.; G.P., K. Effect of Concentration on the Polarizability of Isopropyl Alcohol Indimethyl Sulfoxide. Zh.Fiz.Khim. 1982, 56, 867-870.

18. Zhang, J.; Zhang, H.; Wu, T.; Wang, Q.; van der Spoel, D. Comparison of Implicit and Explicit 
Solvent Models for the Calculation of Solvation Free Energy in Organic Solvents. J. Chem. Theory Comput. 2017, 13, 1034-1043.

19. Ma, B.; Nussinov, R. Molecular Dynamics Simulations of Alanine Rich Beta-Sheet Oligomers: Insight into Amyloid Formation. Protein Sci. 2002, 11, 2335-2350.

20. Sitkoff, D.; Sharp, K. A.; Honig, B. Accurate Calculation of Hydration Free Energies Using Macroscopic Solvent Models. J. Phys. Chem. 1994, 98, 1978-1988.

21. Levy, R. M.; Zhang, L. Y.; Gallicchio, E.; Felts, A. K. On the Nonpolar Hydration Free Energy of Proteins: Surface Area and Continuum Solvent Models for the Solute-Solvent Interaction Energy. J. Am. Chem. Soc. 2003, 125, 9523-9530.

22. Rajamani, S.; Truskett, T. M.; Garde, S. Hydrophobic Hydration from Small to Large Lengthscales: Understanding and Manipulating the Crossover. Proc. Natl. Acad. Sci. U.S.A. 2005, 102, 9475-9480.

23. Tan, C.; Tan, Y.-H.; Luo, R. Implicit Nonpolar Solvent Models. J. Phys. Chem. B 2007, 111, 12263-12274.

24. Tao, K.; Chen, Y.; Orr, A. A.; Tian, Z.; Makam, P.; Gilead, S.; Si, M.; Rencus-Lazar, S.; Qu, S.; Zhang, M.; Tamamis, P.; Gazit, E., Enhanced Fluorescence for Bioassembly by EnvironmentSwitching Doping of Metal Ions. Adv. Funct. Mater. 2020, 1909614.

25. Xue, L.; Liu, C.; Jiang, H. A Ratiometric Fluorescent Sensor with a Large Stokes Shift for Imaging Zinc Ions in Living Cells. Chem. Comm. 2009, 1061-1063. 